\title{
A quadrilateral in the Asaeda-Haagerup category
}

\author{
Marta Asaeda and Pinhas Grossman
}

\begin{abstract}
We construct a noncommuting quadrilateral of factors whose upper sides are each the Asaeda-Haagerup subfactor with index $\frac{5+\sqrt{17}}{2}$ by showing the existence of a $Q$-system in the Asaeda-Haagerup category with index $\frac{7+\sqrt{17}}{2}$. We also conjecture the existence of a $Q$-system in the same category with index $\frac{9+\sqrt{17}}{2}$ and an associated quadrilateral whose upper sides have index $\frac{7+\sqrt{17}}{2}$.
\end{abstract}

Mathematics Subject Classification (2010). 46L37

Keywords. Subfactors, fusion categories.

\section{Introduction}

Subfactor theory was initiated by Jones as a noncommutative Galois theory [10]. It is therefore natural to study the lattice of intermediate subfactors of a finite-index subfactor as a quantum analogue of the subgroup lattice of a finite group. The problem of classifying lattices of intermediate subfactors was posed by Watatani [17], and recent progress has been made by $\mathrm{Xu}$ [18].

The simplest nontrivial lattice is a single proper intermediate subfactor $N \subset P \subset$ $M$. Such inclusions were studied by Bisch and Jones, and they provided a generic construction in terms of the index parameters $[M: P]$ and $[P: N]$, see [3]. Their construction is a free composition, in the sense that the $(P-P)$-bimodules coming from $N \subset P$ and $P \subset M$ have free relations; their results show that there is no obstruction in this case.

The next simplest case is a pair of distinct intermediate subfactors:

$$
\begin{array}{lll}
P & \subset & M \\
\cup & & \cup \\
N & \subset & Q .
\end{array}
$$

Such a configuration is called a quadrilateral of factors if $P \vee Q=M$ and $P \wedge Q=N$. The presence of an additional intermediate subfactor means that we are no longer in 
a free situation. An important notion is commutativity, which means that the tracepreserving conditional expectations of $M$ onto $P$ and $Q$ commute. There is also the dual notion of cocommutativity. Commuting, cocommuting quadrilaterals may be constructed via a tensor product, but it turns out that noncommutativity imposes a great deal of rigidity. Sano and Watatani studied noncommuting quadrilaterals of factors and introduced the notion of angles between subfactors, a numerical invariant which measures the noncommutativity [16].

In [6], the second named author and Jones studied noncommuting quadrilaterals of factors whose sides are supertransitive, a minimality condition which means that the planar algebras are generated by Temperley-Lieb diagrams. They found that there are only two examples of such quadrilaterals up to isomorphism of the planar algebra, a cocommuting quadrilateral coming from an outer action of $S_{3}$ on a factor, with $2=[M: P]=[P: N]-1$ and a noncocommuting quadrilateral with $[M: P]=$ $[P: N]=2+\sqrt{2}$. In [5], the second named author and Izumi showed that if the sides are only required to be 3 -supertransitive, then one still has $[M: P]=[P: N]$ for noncocommuting quadrilaterals and $[M: P]=[P: N]-1$ for cocommuting quadrilaterals (in fact all that is required is that the sides are 2-supertransitive and $N \subset$ $P$ has trivial second cohomology in the sense of Izumi and Kosaki [9]). In the latter case, one has the Galois group $\operatorname{Gal}(M / N) \subseteq S_{3}$, with equality only for the fixed point subfactor of an outer action of $S_{3}$ on a factor. Moreover, if $\{e\} \subset \operatorname{Gal}(M / N) \subset S_{3}$, then one has the following relation among the $(P-P)$-bimodules of the quadrilateral: ${ }_{P} P_{N} \otimes_{N}{ }_{N} P_{P} \cong{ }_{P} P_{P} \oplus\left({ }_{P} M_{M} \otimes_{M \alpha(M)} M_{M} \otimes_{M} M_{M} M_{P}\right)$, where $\alpha$ is an outer automorphism of $M$. In sector notation, this relation is $[\iota \bar{l}]=\left[\operatorname{Id}_{P}\right] \oplus[\bar{\kappa} \alpha \kappa]$, where $\iota={ }_{P} P_{N}$ and $\kappa={ }_{M} M_{P}$.

Subfactors with index less than 4 must have index $4 \cos ^{2} \frac{\pi}{k}$ by Jones' index theorem [10]. The principal graphs were classified by Ocneanu as type $A_{n}, D_{2 n}, E_{6}$, and $E_{8}$ Dynkin diagrams. Note that these are all finite graphs, a condition called finite depth. Principal graphs of subfactors with index 4 have been classified as certain extended Dynkin diagrams; some of these are infinite [15]. In [1], the first named author and Haagerup constructed two exotic finite-depth subfactors with indices $\frac{5+\sqrt{13}}{2}$ (known as the Haagerup subfactor) and $\frac{5+\sqrt{17}}{2}$ (known as the Asaeda-Haagerup subfactor). Along with the recently constructed [2] extended Haagerup subfactor, these (and their duals) are the only finite-depth subfactors with indices strictly between 4 and $3+\sqrt{3}$, see [7].

In [5], all noncommuting, irreducible quadrilaterals with sides of index less than or equal to four were classified, up to isomorphism of the planar algebra; there are seven such quadrilaterals. Moreover, it was shown that the Haagerup subfactor appears as the upper sides of both types of quadrilaterals: there is a noncommuting, noncocommuting quadrilateral all of whose sides are Haagerup subfactors; and there is also a noncommuting, but cocommuting quadrilateral whose upper sides are the Haagerup subfactor but whose lower sides have index $\frac{7+\sqrt{13}}{2}$. This quadrilateral has Galois group $\mathbb{Z} / 3 \mathbb{Z}$, and is in fact the only known example of a noncommuting 
quadrilateral with 2-supertransitive sides and this Galois group.

There was considerable evidence that the Asaeda-Haagerup subfactor should appear in a quadrilateral as well. While it cannot appear in a noncocommuting quadrilateral, it was in fact shown in [5] that any noncommuting but cocommuting quadrilateral which has $\mathbb{Z} / 2 \mathbb{Z}$ Galois group and is maximally supertransitive, in the sense that the upper sides are 5-supertransitive and the lower sides are 3-supertransitive, must have upper sides with principal graph containing the Asaeda-Haagerup graph. Moreover, the candidate principal graph for the lower sides of such an Asaeda-Haagerup quadrilateral, along with two other graphs of the same index, were found independently by Morrison, Peters, and Snyder while searching for possible principal graphs which start off as the Haagerup graph.

The proof of the existence of the cocommuting Haagerup quadrilateral involved showing the existence of a $Q$-system for $\left[\operatorname{Id}_{P}\right] \oplus[\bar{\kappa} \alpha \kappa]$, where $\kappa \bar{\kappa}$ is a $Q$-system for a Haagerup subfactor $P \subset M$, and $\alpha$ is the period 3 automorphism corresponding to the symmetry in the Haagerup graph. The quadrilateral then is obtained by composing these two $Q$-systems. The main technical difficulty was in verifying the $Q$-system relations. This was accomplished through heavy use of the diagrammatic calculus for tensor categories; the diagrams were ultimately evaluated in terms of generators of a Cuntz algebra, using Izumi's construction of the Haagerup subfactor from endomorphisms of a Cuntz algebra [8].

The problem with doing the same thing in the Asaeda-Haagerup category is that there is no corresponding Cuntz algebra representation, so it is difficult to evaluate intertwiner diagrams explicitly. However, there is one principal advantage of the Asaeda-Haagerup category over the Haagerup category: since the graph automorphism has period 2 instead of period 3, the intertwiner equations that occur in the $Q$-system relations are all in 1 dimensional spaces, i.e. they are essentially scalar equations. This allows us to verify the equations by comparing nonzero "states" of the diagrams, rather than fully computing the whole diagrams. The formalism used to express and evaluate these states is very similar to Jones' bipartite graph planar algebra formalism [11].

Once the existence of the "plus one" subfactor is established, its principal graph may be easily computed. The dual graph was given to the authors by Noah Snyder using the subfactor atlas (http://tqft.net/wiki/Atlas_of_subfactors). Interestingly, this dual graph has a symmetry very similar to the original Asaeda-Haagerup graph, leading us to conjecture that the construction may be iterated once more: i.e. there may exist a subfactor in the Asaeda-Haagerup category with index $\frac{9+\sqrt{17}}{2}$ and associated quadrilateral with upper sides having the new Asaeda-Haagerup "plus one" graphs. Checking this conjecture should be straightforward using the methods of this paper combined with methods of [1], but requires some computation. We hope to do this soon.

Aside from the application to classification of quadrilaterals, the existence of the $(\mathrm{AH}+1)$-subfactor should be of independent interest as there is a dearth of finite depth 
subfactors with small index.

The paper is organized as follows: after the present introductory section, Section 2 is a background section reviewing some basic facts about $Q$-systems and biunitary connections. Section 3 proves some identities of intertwiners in the Asaeda-Haagerup category; this is a lot of the workload of the proof of the main theorem. In Section 4 we prove the existence of the Asaeda-Haagerup "plus one" subfactor and the associated quadrilateral.

Acknowledgments. The authors would like to thank Masaki Izumi for conjecturing the existence of the Asaeda-Haagerup quadrilateral, which along with his construction of the Haagerup quadrilateral is the inspiration for the present work; and for many helpful conversations. The authors would like to thank Noah Snyder for helpful comments on the manuscript and for finding the dual graph of the new AsaedaHaagerup "plus one" subfactor with the subfactor atlas.

\section{Preliminaries}

2.1. Subfactors, bimodules, and $Q$-systems. Let $M$ be a Type $\mathrm{II}_{1}$ factor with unique normalized trace tr, and let $1 \in N \subset M$ be a finite-index subfactor. Let $\kappa$ and $\bar{\kappa}$ denote, respectively, the Hilbert space completions of the multiplication bimodules ${ }_{N} M_{M}$ and ${ }_{M} M_{N}$ with respect to tr.

Following sector notation, we will often omit the tensor symbol when writing relative tensor products, so that e.g. $\kappa \bar{\kappa}$ means $\kappa \otimes_{M} \bar{\kappa}$. For any two $(A-B)$-bimodules $\rho$ and $\sigma$, the intertwiner space $\operatorname{Hom}_{A, B}(\rho, \sigma)$ will be denoted by $(\rho, \sigma)$. We have two distinguished bimodules $\operatorname{Id}_{N}={ }_{N} L^{2}(N)_{N}$ and $\operatorname{Id}_{M}={ }_{M} L^{2}(M)_{M}$. Finally, if $\rho$ is an $(A-B)$-bimodule, $\sigma$ is a $(B-C)$-bimodule, and $\lambda$ is a $(C-D)$-bimodule, then the bimodules $(\rho \sigma) \lambda$ and $\rho(\sigma \lambda)$ are naturally isomorphic, and we will think of them as being identified via this isomorphism. Similarly, $\rho, \rho \otimes \operatorname{Id}_{B}$ and $\operatorname{Id}_{A} \otimes \rho$ are naturally isomorphic and we will identify these as well.

We recall Longo's conjugacy theory [12], which was originally formulated for endomorphisms of Type III factors and translated to the finite setting by Masuda [14]. There exist isometries $r_{\kappa} \in\left(\operatorname{Id}_{N}, \kappa \bar{\kappa}\right)$ and $\bar{r}_{\kappa} \in\left(\operatorname{Id}_{M}, \kappa \bar{\kappa}\right)$ satisfying

$$
\left(r_{\kappa}^{*} \otimes \operatorname{Id}_{\kappa}\right) \circ\left(\operatorname{Id}_{\kappa} \otimes \bar{r}_{\kappa}\right)=\frac{1}{d} \operatorname{Id}_{\kappa}
$$

and

$$
\left(\operatorname{Id}_{\bar{\kappa}} \otimes r_{\kappa}^{*}\right) \circ\left(\bar{r}_{\kappa} \otimes \operatorname{Id}_{\bar{\kappa}}\right)=\frac{1}{d} \operatorname{Id}_{\bar{\kappa}},
$$

where $d=[M: N]$ is the Jones index of $N \subset M$.

We will make heavy use of the diagrammatic calculus for tensor categories, in which morphisms are represented by vertices from which emanate strings labeled by the origin objects (upwards) and by the destination objects (downwards). Straight 
strings labeled by objects correspond to identity morphisms, and strings labeled by identity objects are often suppressed. Tensoring is depicted by horizontal concatenation, and composition by vertical concatenation. Diagrams are read from top to bottom.

Then if we let

$$
\bigcap_{\kappa}=\sqrt{d} r_{\kappa} \text { and } \bigcap_{\bar{\kappa}}=\sqrt{d} \bar{r}_{\kappa},
$$

the above equations become
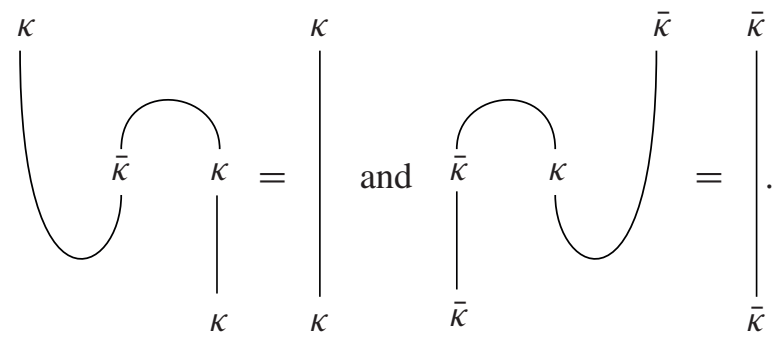

Definition 2.1. A $Q$-system over a $\mathrm{II}_{1}$ factor $N$ is a triple $(\gamma, T, S)$ where $\gamma$ is an $(N-N)$-bimodule with $\operatorname{dim}\left({ }_{N} \gamma\right)=\operatorname{dim}\left(\gamma_{N}\right), T \in\left(\operatorname{Id}_{N}, \gamma\right)$ and $S \in\left(\gamma, \gamma^{2}\right)$ are isometries, and such that

(1) $\left(S \otimes \operatorname{Id}_{\gamma}\right) \circ S=\left(\operatorname{Id}_{\gamma} \otimes S\right) \circ S$, and

(2) $\left(T^{*} \otimes \operatorname{Id}_{\gamma}\right) \circ S=\left(\operatorname{Id}_{\gamma} \otimes T^{*}\right) \circ S=\frac{1}{d} \operatorname{Id}_{\gamma}$ for some $d>0$.

Note that, while the definition in [14] included the additional condition $S S^{*}=$ $\left(S \otimes \operatorname{Id}_{\gamma}\right) \circ\left(\operatorname{Id}_{\gamma} \otimes S^{*}\right)$, this condition was shown to be redundant in [13].

Theorem 2.2 ([12], [14]). If $N \subset M$ is a $\mathrm{II}_{1}$ subfactor, then $\left(\kappa \bar{\kappa}, r_{\kappa}, \mathrm{Id}_{\kappa} \otimes \bar{r}_{\kappa} \otimes \operatorname{Id}_{\bar{\kappa}}\right)$ is a $Q$-system. Conversely, any $Q$-system over $N$ arises in this way for some $M \supset N$.

If $\gamma \cong \operatorname{Id}_{N} \oplus \sigma$ where $\sigma$ is irreducible, the $Q$-system equations can be simplified; the following result was stated in [5] for infinite factors but is equally true for Type $\mathrm{II}_{1}$ factors.

Proposition 2.3. Let $\sigma$ be a self-conjugate $(N-N)$-bimodule such that $\operatorname{dim}\left({ }_{N} \sigma\right)=$ $\operatorname{dim}\left(\sigma_{N}\right)=d$ and $\sigma \not \operatorname{Id}_{N}$. Then $\operatorname{Id}_{N} \oplus \sigma$ admits a $Q$-system if and only if there exist isometries $R \in\left(\operatorname{Id}_{N}, \sigma^{2}\right)$ and $S \in\left(\sigma, \sigma^{2}\right)$ such that

(1) $\left(S \otimes \operatorname{Id}_{\sigma}\right) \circ R=\left(\operatorname{Id}_{\sigma} \otimes S\right) \circ R$, and

(2) $\frac{\sqrt{d+1}}{d}\left(R \otimes \operatorname{Id}_{\sigma}-\operatorname{Id}_{\sigma} \otimes R\right)=\left(\operatorname{Id}_{\sigma} \otimes S\right) \circ S-\left(S \otimes \operatorname{Id}_{\sigma}\right) \circ S$. 
In pictures, if we set

$$
\bigcap_{\sigma}=\sqrt{d} R \text { and } \overbrace{\sigma}^{\sigma}=d^{\frac{1}{4}} S,
$$

then this becomes

(1)

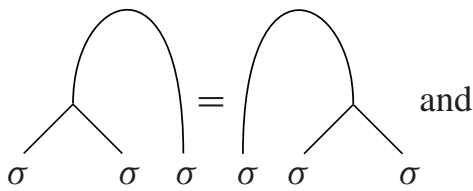

(2)

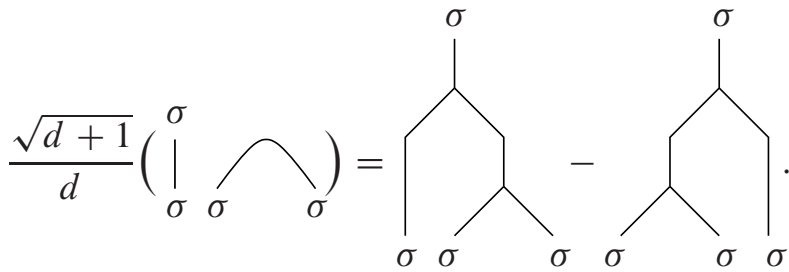

2.2. Connections and bimodules. Let $N \subset M$ be a finite index subfactor. The even vertices in the principal (respectively, dual) graph corresponded to the irreducible $N-N(M-M)$-bimodules which occur in the decomposition of the tensor powers of $\kappa \bar{\kappa}(\bar{\kappa} \kappa)$, and the odd vertices to the $N-M(M-N)$-bimodules which occur in the decomposition of the even bimodules tensored again on the right by $\kappa(\bar{\kappa})$, where $\kappa$ as before is the completion of ${ }_{N} M_{M}$.

To handle bimodules concretely, we use Ocneanu's paragroup theory. If $N \subset M$ has finite depth, the $(N-N)$-bimodules [resp. $(N-M)$-bimodules] may be represented as biunitary connections whose horizontal graphs are both the principal graph (resp. whose upper graph is the principal graph and whose lower graph is the dual graph) of $N \subset M$.

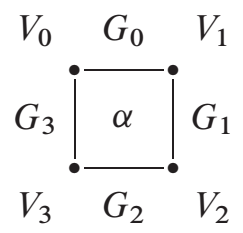

Figure 1. Schematic representation of a connection; cells are based loops around the four graphs.

In this formalism, direct sums of bimodules are then given by merging the vertical graphs as a disjoint union, and tensor products are given by composing the vertical graphs, and the connection accordingly. For more details, we refer the reader to [4] and $\S 3$ of [1]. 
The connection may be extended linearly to cells composed of formal linear combinations of edges, i.e. elements of the Hilbert spaces associated to each pair of vertices with orthonormal basis indexed by the edges between those vertices. One can then define gauge transformations between two biunitary connections on the same graphs - see $\$ 3$ of [1] for details. We recall the following result from Theorem 3 in [1].

Theorem 2.4. A vertical gauge transformation between two biunitary connections on the same graphs gives an isomorphism between the associated bimodules. Conversely, if two bimodules given by biunitary connections with the same horizontal graphs are isomorphic, then the vertical graphs are also identical and the bimodule isomorphism is given by a vertical gauge transformation.

Similarly, it follows from Ocneanu compactness (as in the proof of the above theorem in [1]) that any intertwiner between two bimodules represented by biunitary connections with the same horizontal graphs can be expressed as a map on the vertical edge spaces. Such intertwiners then compose the same way as maps, and act linearly componentwise on composite edges in tensor products. By a slight abuse of notation, we will often identify bimodules with their associated connections in the sequel.

\section{Intertwiners in the Asaeda-Haagerup category}

In [1], the first-named author and Haagerup constructed a subfactor $N \subset M$ with index $\frac{5+\sqrt{17}}{2}$. The principal graph is

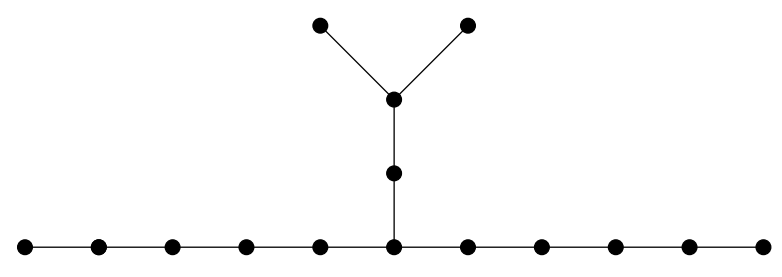

and the dual graph is

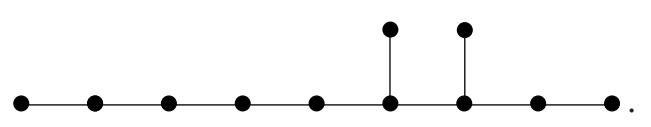

They explicitly computed the biunitary connection for $\kappa={ }_{N} L^{2}(M)_{M}$. For future reference we include the four graphs of $\kappa$ : 


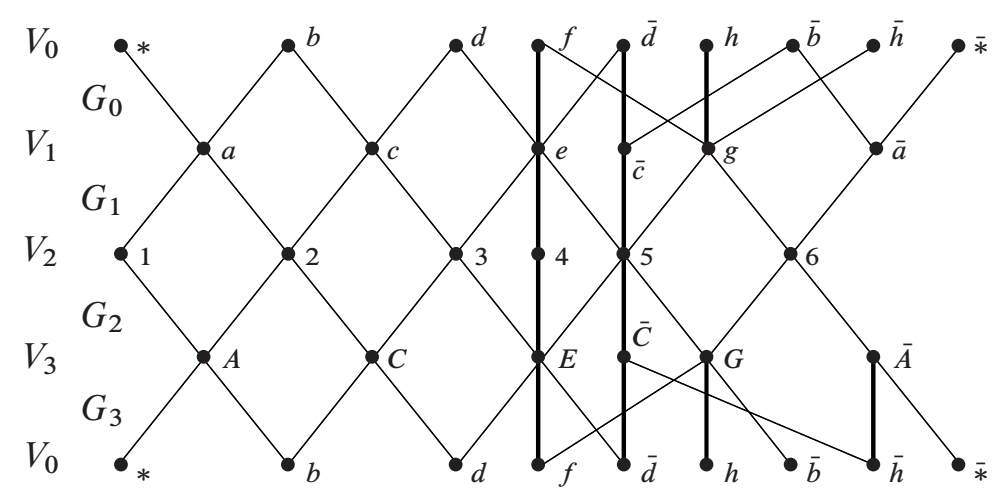

We imagine these four graphs as being wrapped in a square, so that the first and third graphs from the top are the "horizontal" graphs and the second and fourth are the "vertical" graphs in the square.

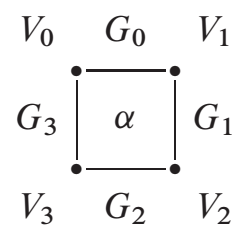

Figure 2. Follow clockwise from top left to get the vertical picture.

Then composing with the dual gives the graphs of $\kappa \bar{\kappa}$ (note that the left vertical graph $G_{3}$ is "upside down", so we reflect vertically before composing):

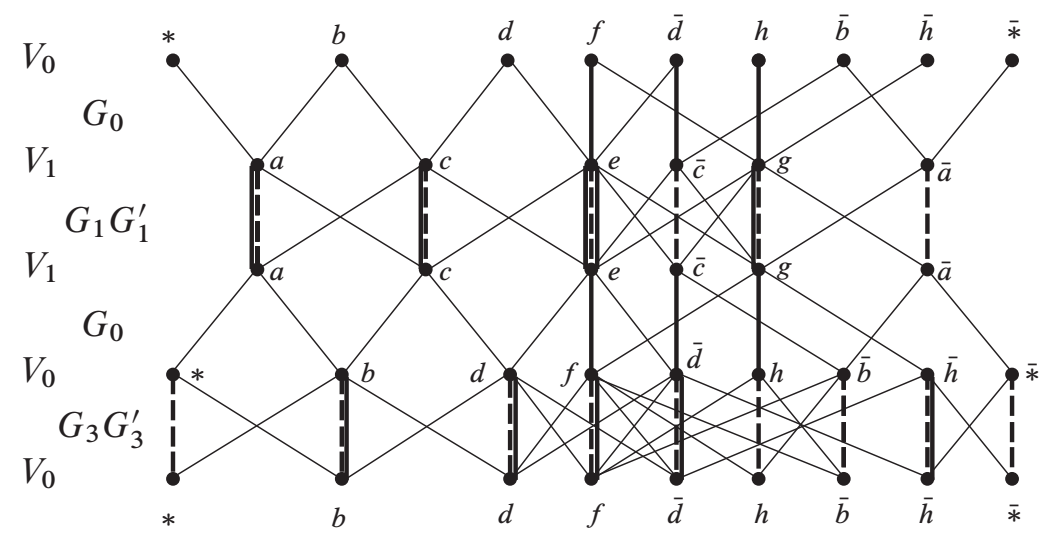

The connection $\kappa \bar{\kappa}$ decomposes into $\mathrm{Id}_{N}$, whose vertical graphs are indicated by the dotted lines, and whose value on every cell is 1 , and another connection, which we 
will call $\rho$. The vertical graphs for $\rho$ are given by the complements of the dotted lines in the graphs of $\kappa \bar{\kappa}$; the connection is determined only up to vertical gauge choice and a representative, which we will take as well, was computed in Tables 8 and 9 in [1]. We will call the connection corresponding to the vertexsymmetric with respect to $\operatorname{Id}_{N}$ in the principal graph $\alpha$; it is a 1-dimensional $(N-N)$-bimodule. The vertical graphs of $\alpha$ switch $x$ with $\tilde{x}$ for each $x$ (we take $\tilde{\tilde{x}}=x$ for all $x$ and $\tilde{y}=y$ for those vertices $y$ which do not have any labeled " $\tilde{y}$ ").

The principal graph can then be labeled by bimodules as follows:

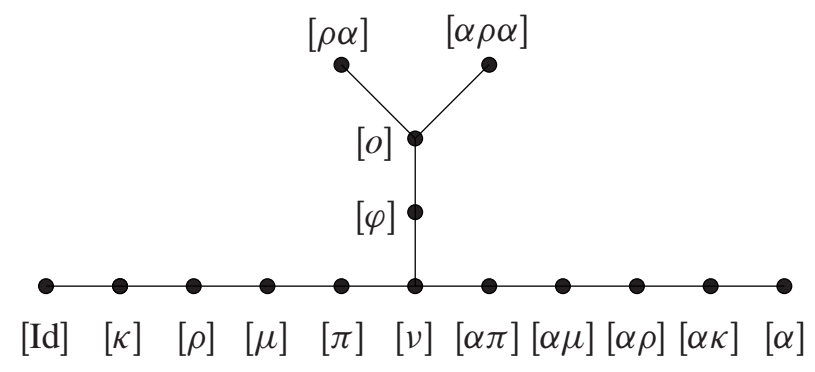

Following the sector notation, the square brackets denote isomorphism classes. The fact that $[\alpha \rho] \neq[\rho \alpha]$ implies that the $(M-M)$-bimodule $\bar{\kappa} \alpha \kappa$ is irreducible: we have $\operatorname{dim}(\bar{\kappa} \alpha \kappa, \bar{\kappa} \alpha \kappa)=\operatorname{dim}(\alpha \kappa \bar{\kappa}, \alpha \kappa \bar{\kappa})=\operatorname{dim}\left(\operatorname{Id}_{N} \oplus \alpha \rho, \operatorname{Id}_{N} \oplus \rho \alpha\right)=1$. Also note the fusion rule $[\rho \alpha \rho]=[\alpha \rho \alpha] \oplus[\eta]$. This implies that $\operatorname{dim}\left(\bar{\kappa} \alpha \kappa,(\bar{\kappa} \alpha \kappa)^{2}\right)=1$.

We want to fix certain intertwiners. Recall that an intertwiner between two connections with the same horizontal graphs is given by a collection of maps on the vertical edge spaces. To describe such an intertwiner, we list the maps corresponding to each edge in the vertical graphs of the origin of the intertwiner.

We use the following notation. Each edge will be denoted by the pair of vertices it connects, e.g. " $* A$ " denotes the edge in the left vertical graph of $\kappa$ which connects * to $A$. In principle there could be multiple such edges but in our computations all the edges will be simple. Composite edges will be denoted by the vertices from each component, e.g. " $* A b$ " denotes the edge in the left vertical graph of $\kappa \bar{\kappa}$ which is composed of the edges $* A$ in $\kappa$ and $A b$ in $\bar{\kappa}$. Finally, each gauge map will be represented as an edge of the origin mapping to a linear combination of edges in the destination.

The intertwiners $r_{\kappa}$ and $\bar{r}_{\kappa}$ are determined up to a scalar. We can fix the scalar by assigning a complex unitary as the gauge entry corresponding to any simple edge in $\kappa \bar{\kappa}$. So we fix $* * \mapsto * A *$ and then $r_{\kappa}$ can be computed using the methods of [1], see pp. 40-43. We also use the following notation of [1]:

$$
\beta=\sqrt{\frac{5+\sqrt{17}}{2}}, \quad \beta_{n}=\sqrt{\beta^{2}-n}, \quad \beta_{n}^{\prime}=\beta_{n}^{2}, \quad \gamma=\sqrt{2 \beta^{2}-1}, \quad \gamma^{\prime}=\gamma^{2} .
$$

Then the map $r_{k}$ is defined by the following table. 


\begin{tabular}{|l|l|}
$* * \mapsto * A *$ & $a a \mapsto \frac{1}{\beta} a 1 a+\frac{\beta_{1}}{\beta} a 2 a$ \\
$b b \mapsto \frac{1}{\beta_{1}} b A b+\frac{\beta_{2}}{\beta_{1}} b C b$ & $c c \mapsto \frac{\beta_{1}}{\beta \beta_{2}} c 2 c+\frac{\beta_{1}}{\sqrt{2} \beta_{2}} c 3 c$ \\
$d d \mapsto \frac{2}{\beta_{1}^{2}} d C d+\frac{2 \sqrt{2}}{\beta \beta_{2}} d E d$ & $e e \mapsto \frac{\beta_{2}}{2 \sqrt{2}} e 3 e+\frac{1}{\beta} e 4 e+\frac{\beta_{2}}{\beta_{-1}} e 5 e$ \\
$f f \mapsto\left(\frac{1}{2}+\frac{\sqrt{2}}{\beta \beta-1}\right) f E f+\frac{1}{\beta_{2}} f G f$ & $\tilde{c} \tilde{c} \mapsto \tilde{c} 5 \tilde{c}$ \\
$\tilde{d} \tilde{d} \mapsto \frac{2 \sqrt{2}}{\beta \beta_{2}} \tilde{d} E \tilde{d}+\frac{2}{\beta_{1}^{2}} \tilde{d} \tilde{C} \tilde{d}$ & $g g \mapsto \frac{\beta_{2}}{\beta_{1}} g 5 g+\frac{1}{\beta_{1}} g 6 g$ \\
$h h \mapsto h G h$ & $\tilde{a} \tilde{a} \mapsto \tilde{a} G \tilde{a}$ \\
$\tilde{b} \tilde{b} \mapsto \tilde{b} G \tilde{b}$ & \\
$\tilde{h} \tilde{h} \mapsto \frac{\beta_{2}}{\beta_{1}} \tilde{h} \tilde{C} \tilde{h}+\frac{1}{\beta_{1}} \tilde{h} \tilde{A} \tilde{h}$ & \\
$\tilde{*} \tilde{*} \mapsto \tilde{*} \tilde{A}$ & \\
\hline
\end{tabular}

Similarly, $\bar{r}_{\kappa}$ can be fixed by setting $11 \mapsto 1 a 1$, and then we get the following table for $\bar{r}_{\kappa}$.

\begin{tabular}{|c|c|}
$A A \mapsto \frac{1}{\beta} A * A+\frac{\beta_{1}}{\beta} A b A$ & $11 \mapsto 1 a 1$ \\
$C C \mapsto \frac{\beta_{1}}{\beta \beta_{2}} C b C+\frac{\beta_{1}}{\sqrt{2} \beta_{2}} C d C$ & $22 \mapsto \frac{1}{\beta_{1}} 2 a 2+\frac{\beta_{2}}{\beta_{1}} 2 c 2$ \\
$E E \mapsto \frac{\beta_{1}}{\sqrt{2} \beta_{-1}} E d E+\frac{\beta_{1}}{\sqrt{2} \beta_{-1}} E \tilde{d} E$ & $33 \mapsto \frac{\beta_{1}}{\sqrt{2} \beta \beta_{2} \beta_{2}^{\prime}}\left(\beta_{1}^{\prime}+\frac{1}{\gamma^{\prime}}\right) 3 c 3$ \\
$\quad+\frac{\sqrt{2}}{\beta-1} E f E$ & $+\frac{\sqrt{2} \beta \beta_{1}^{\prime}}{\beta_{2} \gamma^{\prime}} 3 e 3$ \\
$\widetilde{C} \widetilde{C} \mapsto \frac{\sqrt{2} \gamma}{\beta_{1} \beta_{2}^{\prime}} \widetilde{C} \tilde{d} \tilde{C}+\frac{\sqrt{2}}{\beta_{2}^{\prime}} \tilde{C} \tilde{h} \widetilde{C}$ & $44 \mapsto 4 e 4$ \\
$G G \mapsto \frac{\sqrt{2}}{\beta_{1}} G f G+\frac{1}{\beta} G \tilde{b} G$ & $55 \mapsto \frac{\beta_{-1}^{\prime}}{\sqrt{2} \beta_{2} \beta_{1}^{\prime}} 5 e 5+\frac{\beta_{1}}{\sqrt{2} \gamma} 5 \tilde{c} 5$ \\
$\quad+\frac{1}{\beta} G h G$ & $+\frac{\sqrt{2}}{\beta_{2}^{\prime}} 5 g 5$ \\
$\tilde{A} \tilde{A} \mapsto \frac{\sqrt{2}}{\beta_{2}} \tilde{A} \tilde{h} \tilde{A}+\frac{1}{\beta} \tilde{A} \tilde{*} \tilde{A}$ & $66 \mapsto \frac{\sqrt{2}}{\beta_{2}} 6 g 6+\frac{1}{\beta} 6 \tilde{a} 6$ \\
\hline
\end{tabular}

We need to check that the these choices for $r_{\kappa}$ and $\bar{r}_{\kappa}$ are consistent.

Lemma 3.1. The intertwiners $r_{\kappa}$ and $\bar{r}_{\kappa}$ defined as above, satisfy the conjugacy Equations 1 and 2.

Proof. Since $\operatorname{dim}\left(\operatorname{Id}_{N}, \kappa \bar{\kappa}\right)=\operatorname{dim}\left(\operatorname{Id}_{M}, \bar{\kappa} \kappa\right)=1$ and $r_{\kappa}, \bar{r}_{\kappa}$ are isometries, Equations 1 and 2 are satisfied up to a unitary scalar. Since all the nonzero entries of the intertwiners are positive, that scalar must be 1 .

The connection for $\rho$ was chosen in [1] so that all the gauge unitaries between $\rho$ and $\kappa \bar{\kappa}$ corresponding to simple edges between distinct vertices are the same. Let $v: \rho \rightarrow \kappa \bar{\kappa}$ be the isometry determined by fixing those gauge unitaries to be 1 . We will need some coefficients of $v$. For edges $x y \in \rho, x Z y \in \kappa \bar{\kappa}$ we use the notation $v(x y, x z y)$ for the coefficient of $x Z y$ in the image of $x y$ under $v$. (We will also use similar notation for coefficients of intertwiners of other connections.) 
Lemma 3.2. We have $v(\tilde{h} \tilde{h}, \tilde{h} \tilde{A} \tilde{h})=\frac{\beta_{2}}{\beta_{1}}=-v(b b, b A b)$.

Proof. This is a straightforward computation from the connection tables (see [1], Tables 8 and 9) using the fact that all the simple gauge unitaries corresponding to edges between distinct vertices were chosen to be 1 .

Finally, let $w: \alpha \rho \alpha \kappa \rightarrow \rho \alpha \kappa$ be the isometry constructed in [1], §5. We recall the following coefficients. (Note that while not all of these are explicitly written in [1], they are implicit from the gauge choices made there.)

Lemma 3.3. We have

$$
\begin{aligned}
w(b \tilde{b} h \tilde{h} \tilde{A}, b * \tilde{*} \tilde{A}) & =w(\tilde{h} h f f G, \tilde{h} \tilde{h} h G) \\
& =w(* \tilde{*} \tilde{h} h G, * b \tilde{b} G) \\
& =w(\tilde{*} * b \tilde{b} G, \tilde{*} \tilde{h} h G) \\
& =1 \\
& =-w(b \tilde{b} f f G, b b \tilde{b} G) \\
& =w(\tilde{h} h \tilde{b} b A, \tilde{h} \tilde{*} * A) .
\end{aligned}
$$

Next, we define diagrams with the appropriate normalizations.

Let

$$
\begin{aligned}
& \bigcap_{\kappa}=\sqrt{\beta} r_{\kappa}, \quad \overbrace{\bar{\kappa}}=\sqrt{\beta} \bar{r}_{\kappa}, \\
& =\sqrt{\frac{\beta}{\beta_{1}}} v,
\end{aligned}
$$

Then let
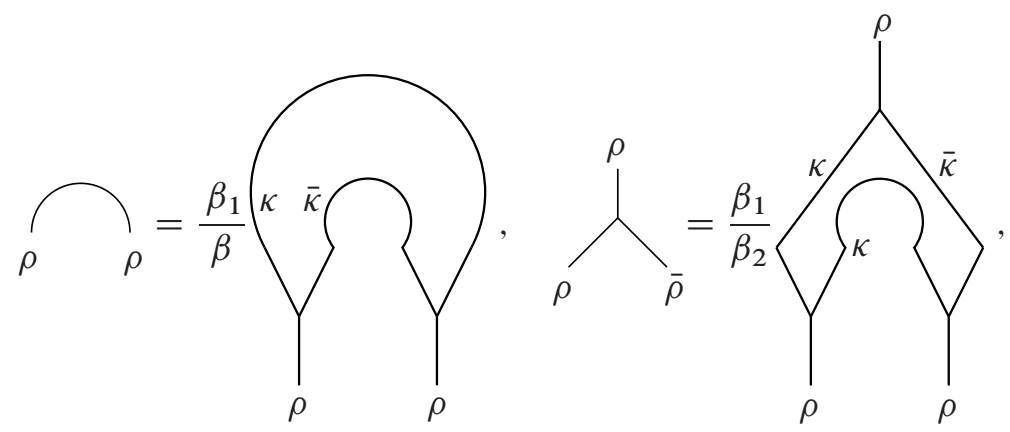
and

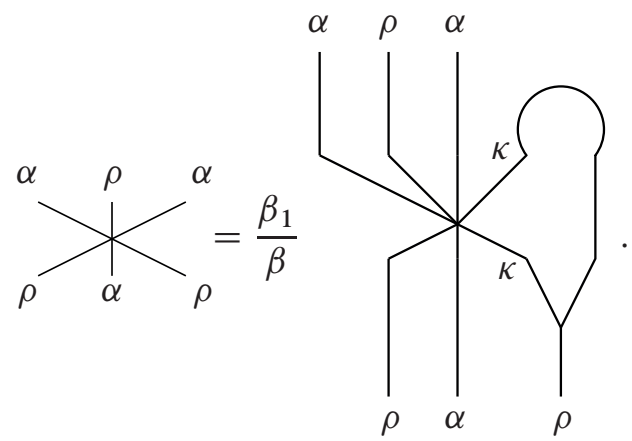

For each of these diagrams let the diagram obtained by rotation by $\pi$ be the adjoint.

Define
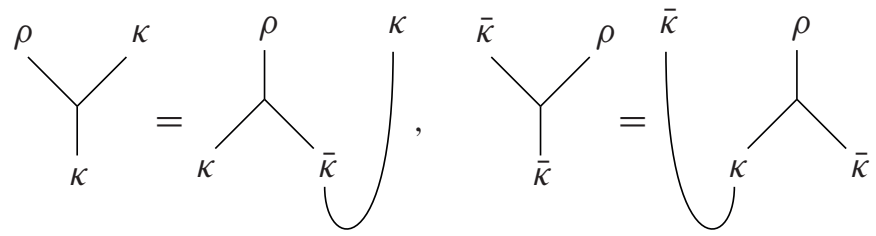

and again let the diagrams obtained by rotation by $\pi$ be the adjoints.

Lemma 3.4. We have
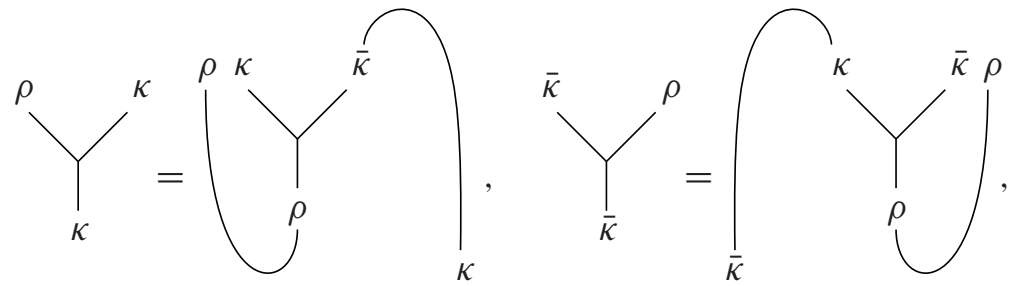

and, similarly,

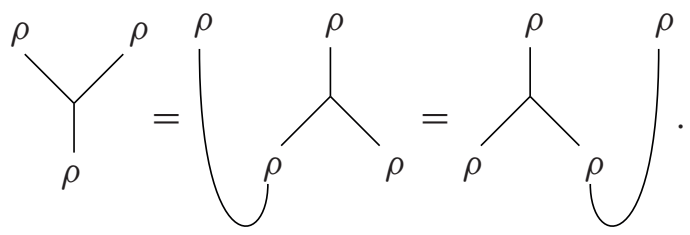

Proof. By Frobenius reciprocity, the two diagrams in the first equation have the same norm (see [5], Lemma 8.1), and they belong to the same one-dimensional space. Consider the edge $* b A$ in $\rho \kappa$; both diagrams send it to $* A$ with positive coefficient, 
so they must represent the same intertwiner. The proofs of the other equalities are similar.

These properties allows us to unambiguously "rotate" these trivalent vertices.

We will need to compute coefficients of more complicated intertwiner diagrams, so we introduce the following formalism. By a vertex of an intertwiner diagram we will mean a crossing or relative extremum of the y-coordinate. The intertwiners represented by the vertices are called the elementary intertwiners of the diagram. Then each intertwiner diagram is a composition of elementary intertwiners tensored with identity morphisms. Such a diagram represents an intertwiner in $\operatorname{Hom}\left(\lambda_{1} \ldots \lambda_{n}, \mu_{1} \ldots \mu_{m}\right)$, where $\lambda_{1}, \ldots, \lambda_{n}$ are the bimodules labeling the strings at the top of the diagram and $\mu_{1}, \ldots, \mu_{m}$ label the strings at the bottom. Since an intertwiner is a map from the edge space of $\lambda_{1} \ldots \lambda_{n}$ to that of $\mu_{1} \ldots \mu_{m}$, the diagram can be evaluated on a specific edge in $\lambda_{1} \ldots \lambda_{n}$ by "following" the edge vertically from top to bottom and composing the actions of the elementary intertwiners.

Definition 3.5. A state on an intertwiner diagram is a labeling of the strings of the diagram by edges in the corresponding bimodules and regions of the diagram by vertices such that each string labels an edge connecting the two adjacent regions. (We imagine that the diagram is bounded by a box so one can not "go around" the top of the strings). A state determines a unique edge at each horizontal cross section of the diagram which does not contain a vertex. The spin factor associated to a vertex is the coefficient of the corresponding elementary intertwiner from the edge directly above it to the edge directly below it. The value of a state is the product of the spin factors of all its vertices.

The following lemma is just an exercise in unraveling the definitions of states, intertwiners diagrams, and connections.

Lemma 3.6. If $x$ is an edge in $\lambda_{1} \ldots \lambda_{n}$ and $y$ is an edge in $\mu_{1} \ldots \mu_{m}$, then the $(x, y)$ coefficient of the intertwiner is the sum of the values of all states whose top horizontal cross section is $x$ and whose bottom horizontal cross section is $y$.

In the state diagrams that follow, the connection associated to each string will have a unique edge between the vertices of the adjacent regions, so we omit the labeling of the strings by edges. To avoid clutter, we also sometimes omit the labeling of the strings by the connections if it is clear from context.

We now compute a bunch of coefficients of various intertwiners that we will need later.

Lemma 3.7. We have the following coefficients:

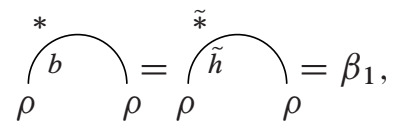

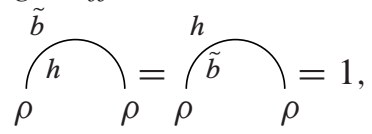


(b) $-\overbrace{\rho}^{*} \int_{\rho}^{\rho} b=\overbrace{\rho}^{\tilde{h}} \widehat{h}_{\rho}^{\rho} \tilde{h}=\beta_{2} \sqrt{\frac{\beta_{1}}{2}}$, $\overbrace{\rho}^{h} \int_{\rho}^{\rho} \tilde{b}=\overbrace{\rho}^{\left.\tilde{b}\right|_{\rho} ^{\rho}}=\sqrt{\frac{\beta_{1}}{2}}$,

(c)

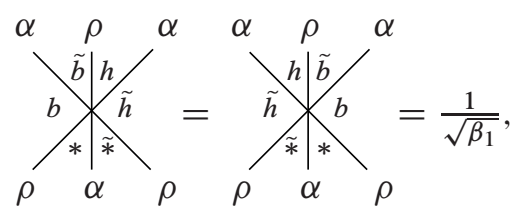

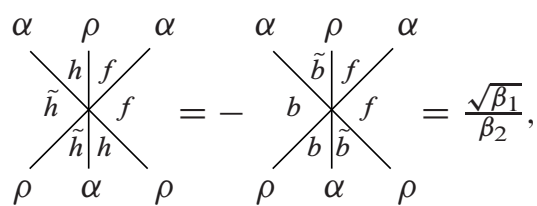

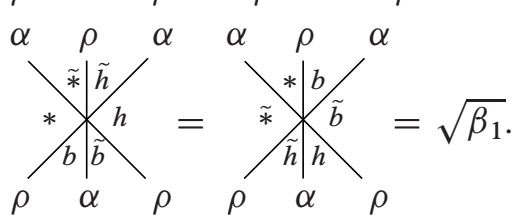

Proof. The idea behind all the computations is the same: we express each intertwiner as a diagram whose elementary intertwiners are all known explicitly. Then the coefficients expand into states which can be evaluated. In principle we have to sum over all states compatible with the coefficient we are computing, but in practice each coefficient will determine a unique state. For each part of the lemma, we illustrate how the state breaks up into elementary intertwiners for the first coefficient computed, and then omit that step for the rest of the coefficients.

(a) We have

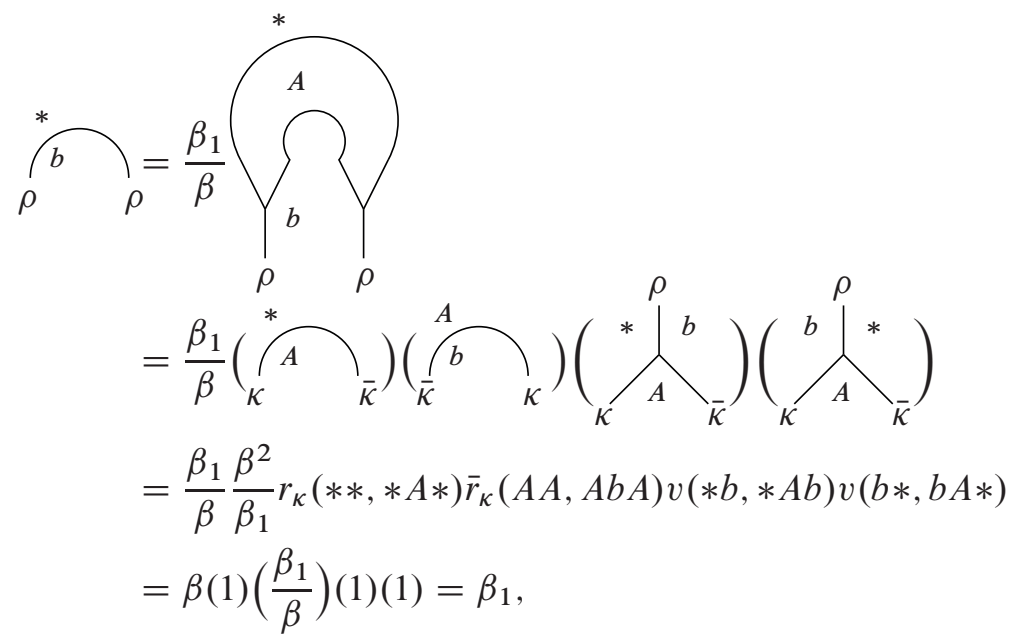



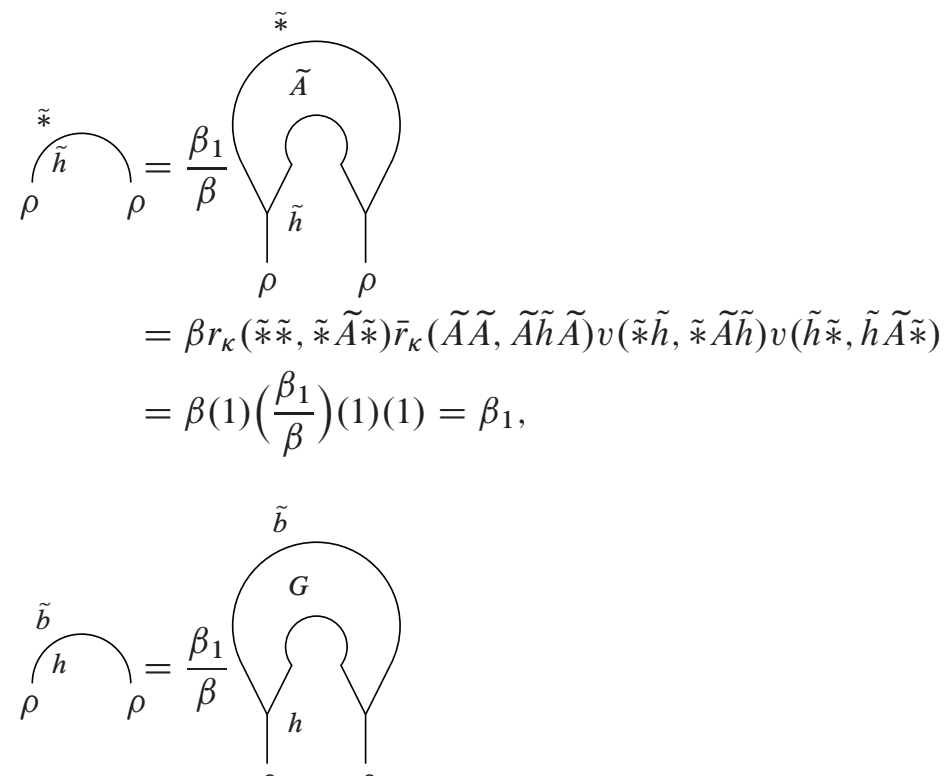

$$
\begin{aligned}
& =\beta r_{\kappa}(\tilde{b} \tilde{b}, \tilde{b} G \tilde{b}) \bar{r}_{\kappa}(G G, G h G) v(\tilde{b} h, \tilde{b} G h) v(h \tilde{b}, h G \tilde{b}) \\
& =\beta\left(\frac{1}{\beta}\right)(1)(1)(1)=1
\end{aligned}
$$

and

$$
\begin{aligned}
\overbrace{\rho}^{h} & =\frac{\beta_{1}}{\beta} \overbrace{\tilde{b}} \\
& =\beta r_{\kappa}(h h, h G h) \bar{r}_{\kappa}(G G, G \tilde{b} G) v(h \tilde{b}, h G \tilde{b}) v(\tilde{b} h, \tilde{b} G h) \\
& =\beta\left(\frac{1}{\beta}\right)(1)(1)(1)=1 .
\end{aligned}
$$

(b) We have

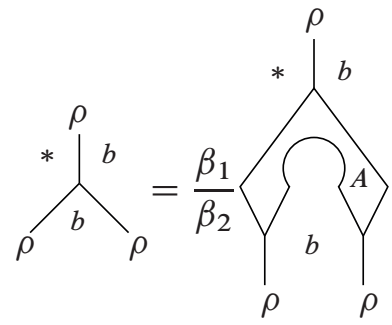




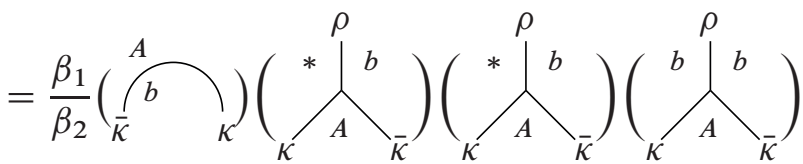

$$
\begin{aligned}
& =\frac{\beta_{1}}{\beta_{2}} \frac{\beta^{2}}{\beta_{1}^{\frac{3}{2}}} \bar{r}_{\kappa}(A A, A b A) v(* b, * A b) v(* b, * A b) v(b b, b A b) \\
& =\beta \sqrt{\frac{\beta_{1}}{2}}\left(\frac{\beta_{1}}{\beta}\right)(1)(1)\left(-\frac{\beta_{2}}{\beta_{1}}\right)=-\beta_{2} \sqrt{\frac{\beta_{1}}{2}},
\end{aligned}
$$

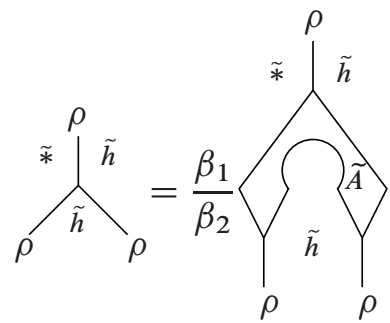

$$
\begin{aligned}
& =\beta \sqrt{\frac{\beta_{1}}{2}} \bar{r}_{\kappa}(\tilde{A} \tilde{A}, \tilde{A} \tilde{h} \tilde{A}) v(\tilde{*} \tilde{h}, \tilde{*} \tilde{A} \tilde{h}) v(\tilde{*} \tilde{h}, \tilde{*} \tilde{A} \tilde{h}) v(\tilde{h} \tilde{h}, \tilde{h} \tilde{A} \tilde{h}) \\
& =\beta \sqrt{\frac{\beta_{1}}{2}}\left(\frac{\beta_{1}}{\beta}\right)(1)(1)\left(\frac{\beta_{2}}{\beta_{1}}\right)=\beta_{2} \sqrt{\frac{\beta_{1}}{2}},
\end{aligned}
$$

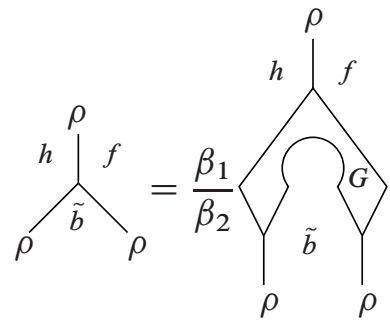

$$
\begin{aligned}
& =\beta \sqrt{\frac{\beta_{1}}{2}} \bar{r}_{\kappa}(G G, G \tilde{b} G) v(h \tilde{b}, h G \tilde{b}) v(\tilde{b} f, \tilde{b} G f) v(h f, h G f) \\
& =\beta \sqrt{\frac{\beta_{1}}{2}}\left(\frac{1}{\beta}\right)(1)(1)(1)=\sqrt{\frac{\beta_{1}}{2}} \text {, }
\end{aligned}
$$


and

$$
\begin{aligned}
& =\beta \sqrt{\frac{\beta_{1}}{2}} \bar{r}_{\kappa}(G G, G h G) v(\tilde{b} h, \tilde{b} G h) v(\tilde{b} f, \tilde{b} G f) v(h f, h G f) \\
& =\beta \sqrt{\frac{\beta_{1}}{2}}\left(\frac{1}{\beta}\right)(1)(1)(1)=\sqrt{\frac{\beta_{1}}{2}} .
\end{aligned}
$$

(c) We have

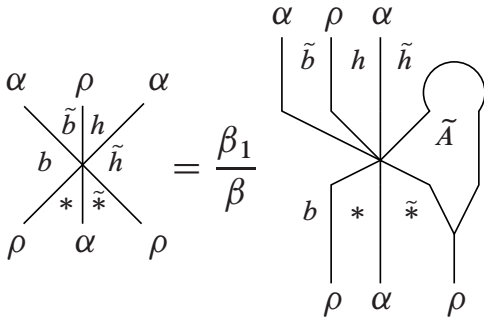

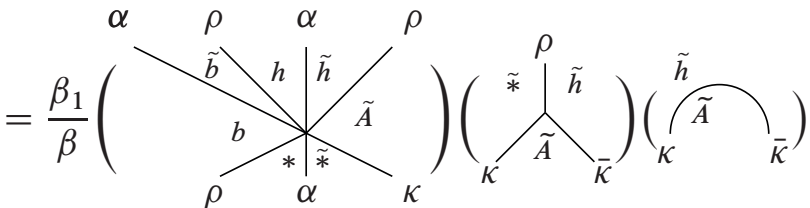

$$
\begin{aligned}
& =\frac{\beta_{1}}{\beta} \frac{\beta}{\sqrt{\beta_{1}}} w(b \tilde{b} h \tilde{h} \tilde{A}, b * \tilde{*} \tilde{A}) v(\tilde{*} \tilde{h}, \tilde{*} \tilde{A} \tilde{h}) r(\tilde{h} \tilde{h}, \tilde{h} \tilde{A} \tilde{h}) \\
& =\sqrt{\beta_{1}}(1)(1)\left(\frac{1}{\beta_{1}}\right)=\frac{1}{\sqrt{\beta_{1}}},
\end{aligned}
$$

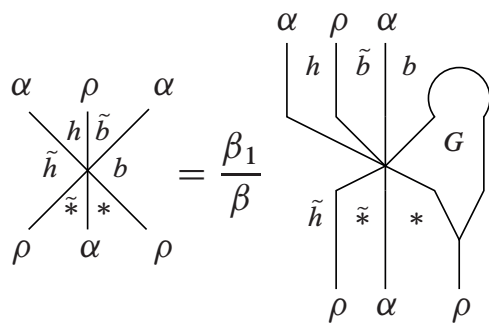




$$
\begin{aligned}
& =\sqrt{\beta_{1}} w(\tilde{h} h \tilde{b} b A, \tilde{h} \tilde{*} * A) v(* b, * A b) r(b b, b A b) \\
& =\sqrt{\beta_{1}}(1)(1)\left(\frac{1}{\beta_{1}}\right)=\frac{1}{\sqrt{\beta_{1}}},
\end{aligned}
$$
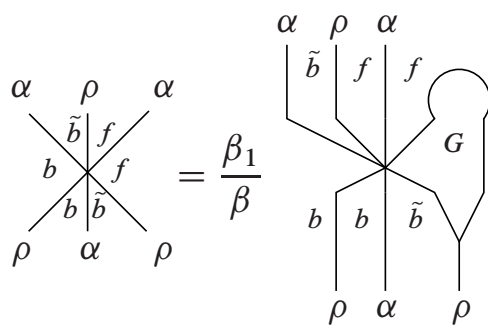

$$
\begin{aligned}
& =\sqrt{\beta_{1}} w(b \tilde{b} f f G, b b \tilde{b} G) v(\tilde{b} f, \tilde{b} G f) r(f f, f G f) \\
& =\sqrt{\beta_{1}}(-1)(1)\left(\frac{1}{\beta_{2}}\right)=-\frac{\sqrt{\beta_{1}}}{\beta_{2}},
\end{aligned}
$$

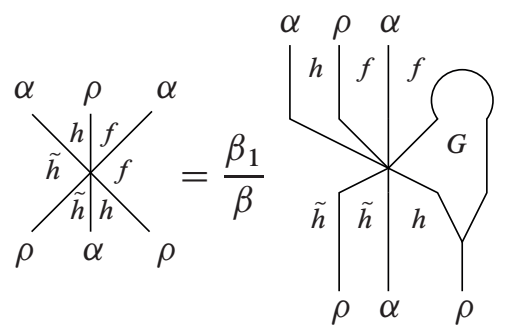

$$
\begin{aligned}
& =\sqrt{\beta_{1}} w(\tilde{h} h f f G, \tilde{h} \tilde{h} h G) v(h f, h G f) r(f f, f G f) \\
& =\sqrt{\beta_{1}}(1)(1)\left(\frac{1}{\beta_{2}}\right)=\frac{\sqrt{\beta_{1}}}{\beta_{2}},
\end{aligned}
$$

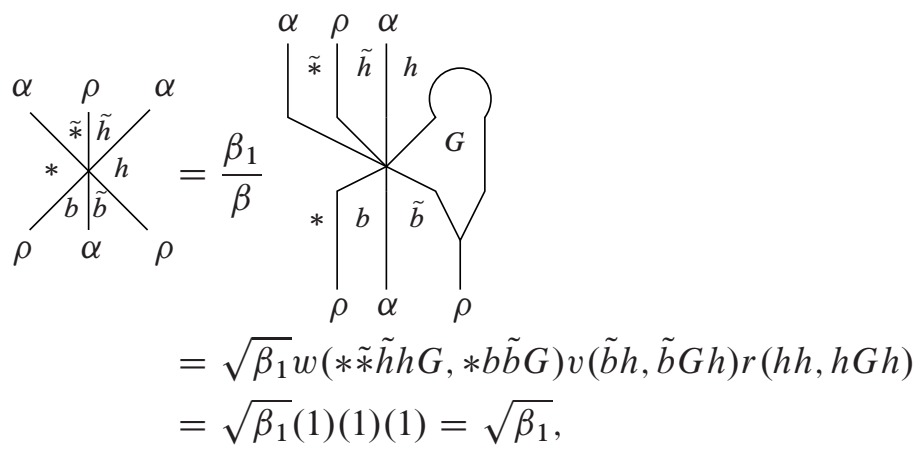


and

$$
\begin{aligned}
\rho_{\alpha}^{\alpha} & =\frac{\beta_{1}}{\beta} \\
& =\sqrt{\beta_{1}} w(\tilde{*} * b \tilde{b} G, \tilde{*} \tilde{h} h G) v(h \tilde{b}, h G \tilde{b}) r(\tilde{b} \tilde{b}, \tilde{b} G \tilde{b}) \\
& =\sqrt{\beta_{1}}(1)(1)(1)=\sqrt{\beta_{1}} .
\end{aligned}
$$

Lemma 3.8. We have
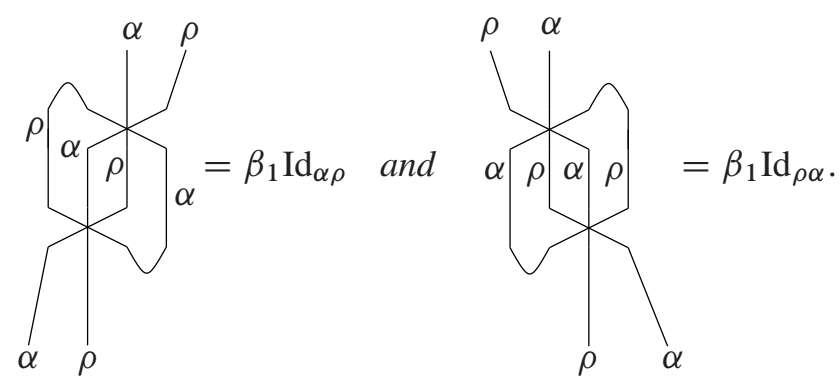

Proof. The left hand side of each equation is a scalar, so we can simply evaluate the unique state compatible with any given edge. For the first equation we have

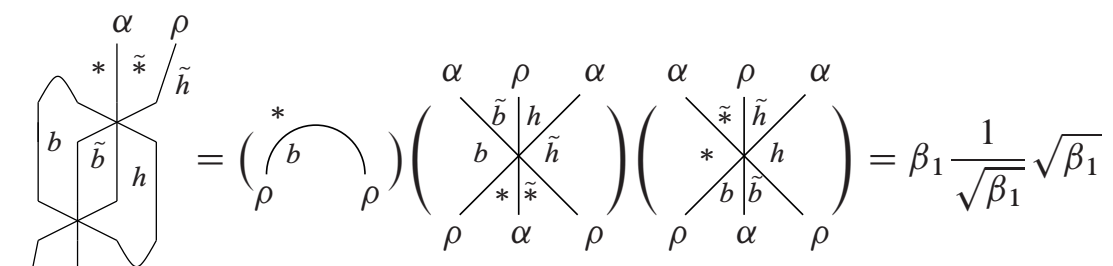

and, for the second one,

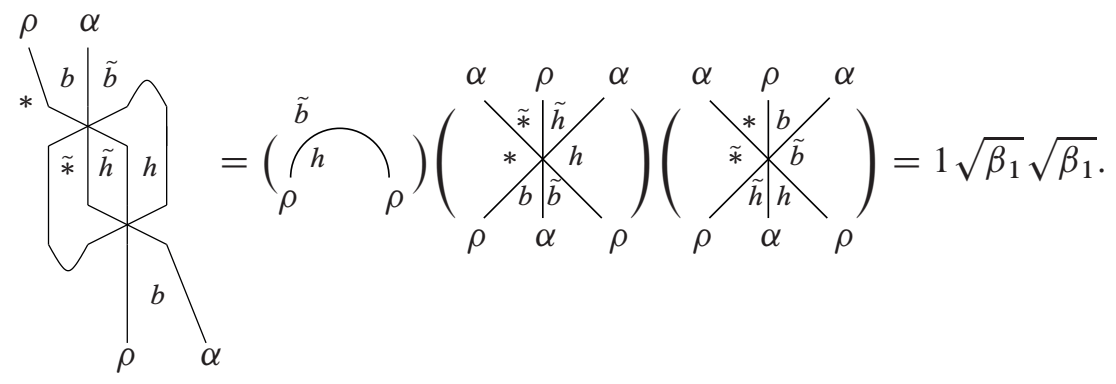


Corollary 3.9. We have

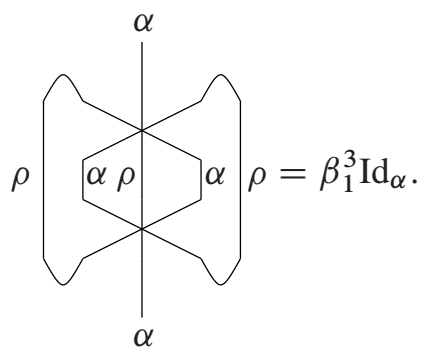

Lemma 3.10. We have

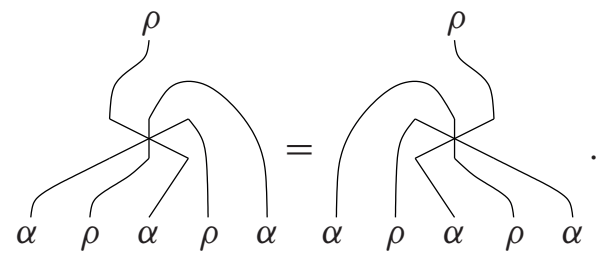

Proof. Since $\operatorname{dim}(\rho, \alpha \rho \alpha \rho \alpha)=1$, we can compare the two sides of the equations using any nonzero coefficient. We choose the coefficient corresponding to the edges $(* b, * \tilde{*} \tilde{h} h \tilde{b} b)$, which admits a unique compatible state for each of the diagrams in the equation. For the left hand side we have

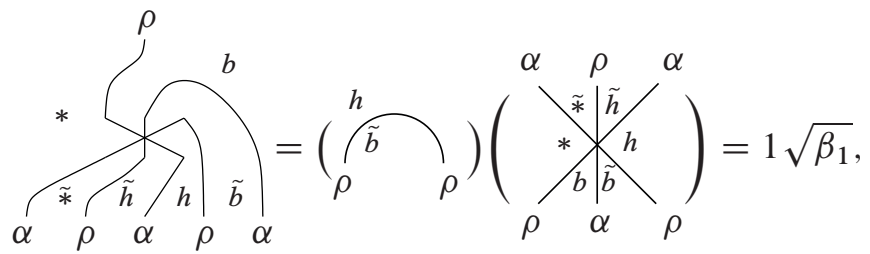

and, for the right hand side,

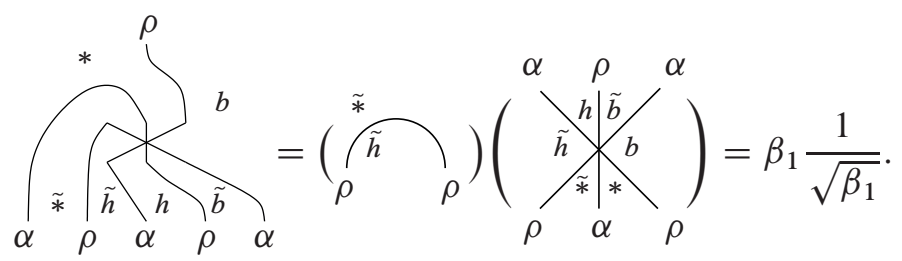


Lemma 3.11. We have

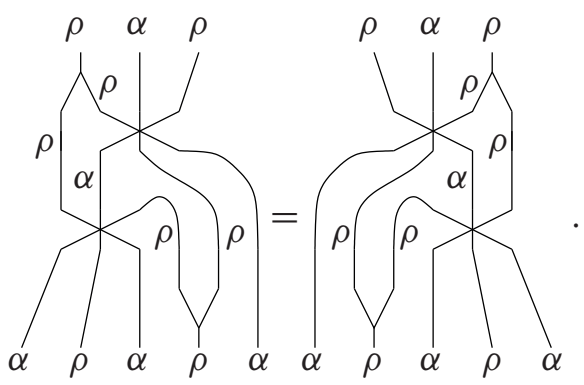

Proof. Again, $\operatorname{dim}(\rho \alpha \rho, \alpha \rho \alpha \rho \alpha)=1$, so we can compare the two diagrams using any nonzero coefficient. We choose $(* b \tilde{b} f, * \tilde{*} \tilde{h} h f f)$, and find, for the left hand side,

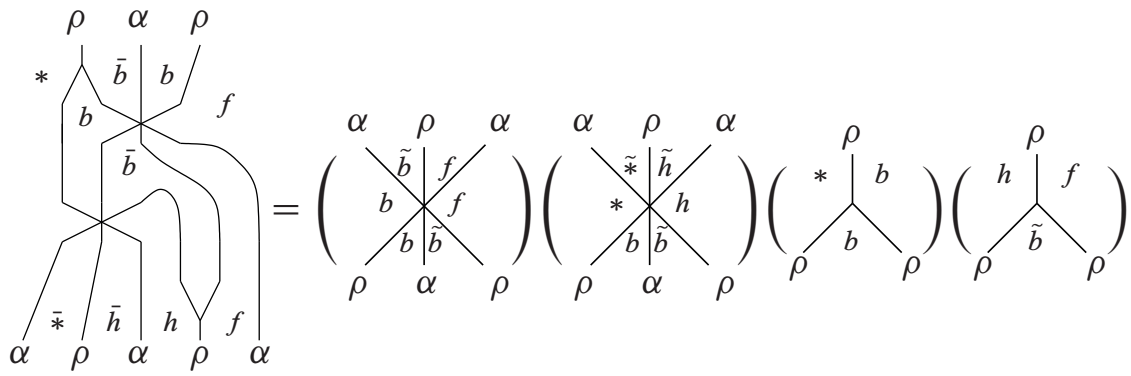

$$
\begin{aligned}
& =-\frac{\sqrt{\beta_{1}}}{\beta_{2}} \sqrt{\beta_{1}}\left(-\beta_{2} \sqrt{\frac{\beta_{1}}{2}}\right) \sqrt{\frac{\beta_{1}}{2}}
\end{aligned}
$$

and, for the right hand side,

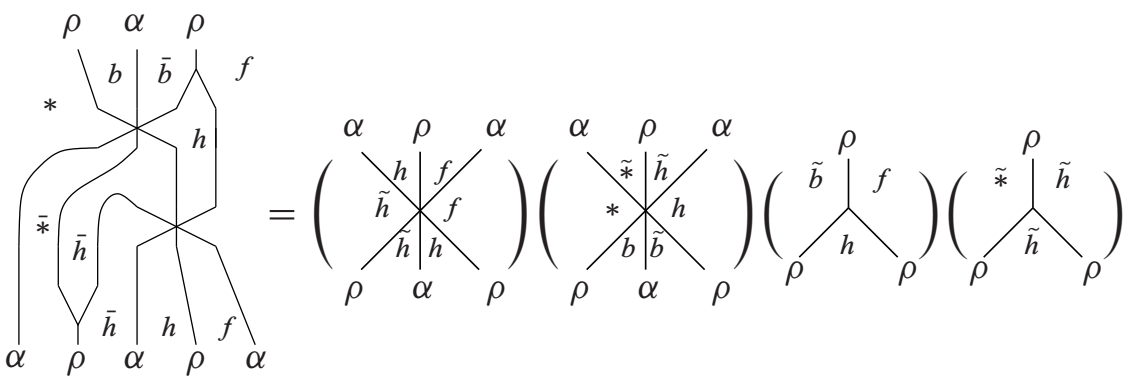

$$
\begin{aligned}
& =\frac{\sqrt{\beta_{1}}}{\beta_{2}} \sqrt{\beta_{1}} \sqrt{\frac{\beta_{1}}{2}}\left(\beta_{2} \sqrt{\frac{\beta_{1}}{2}}\right) .
\end{aligned}
$$


Lemma 3.12. We have

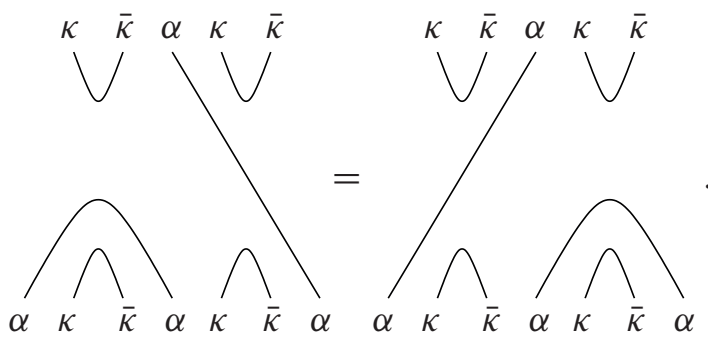

Proof. This time $\operatorname{dim}(\kappa \bar{\kappa} \alpha \kappa \bar{\kappa}, \alpha \kappa \bar{\kappa} \alpha \kappa \bar{\kappa} \alpha)=4$, so evaluating a single nonzero coefficient on each side is insufficient. However, the only compatible states of these diagrams are of the form

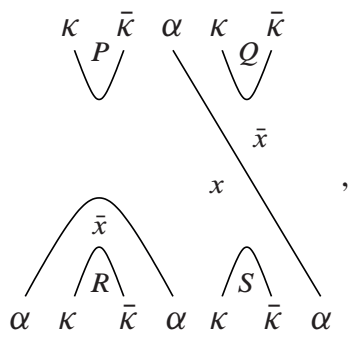

for the left hand side, and

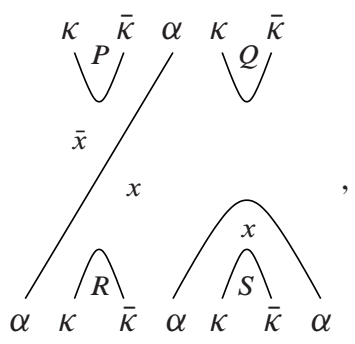

for the right hand side, where $x, P, Q, R, S$ are some vertices from the appropriate graphs.

Since

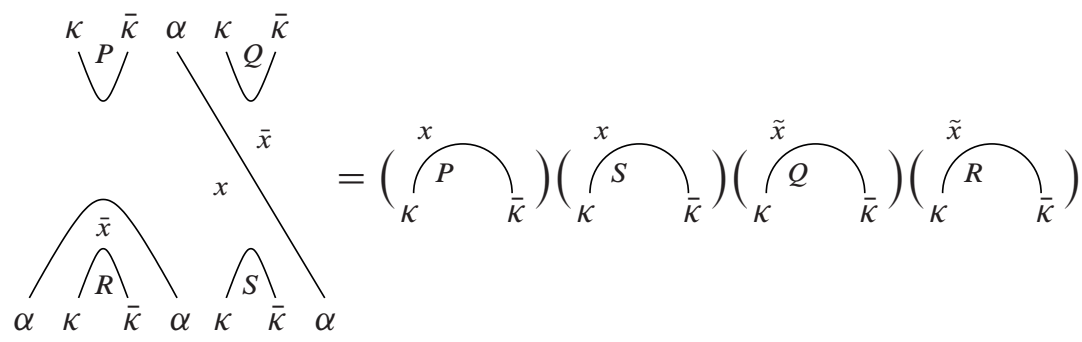




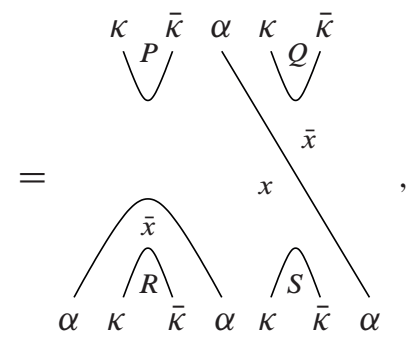

the two intertwiners are the same.

\section{A quadrilateral}

The following lemma was proved for the Haagerup category in [5]. As the proof for the Asaeda-Haagerup category is identical we omit it here.

Lemma 4.1. We have

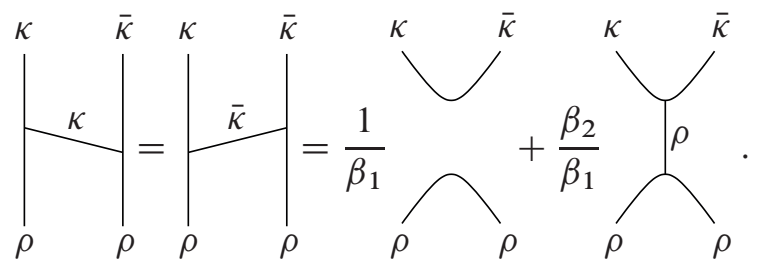

We want to prove that $\operatorname{Id}_{M} \oplus \bar{\kappa} \alpha \kappa$ admits a $Q$-system; to do this we need to show that there are isometries $R \in\left(\operatorname{Id}_{M}, \bar{\kappa} \alpha \kappa \bar{\kappa} \alpha \kappa\right), S \in(\bar{\kappa} \alpha \kappa, \bar{\kappa} \alpha \kappa \bar{\kappa} \alpha \kappa)$ satisfying both (1) and (2) of Proposition 2.3 with $d=\beta^{2}$.

Let
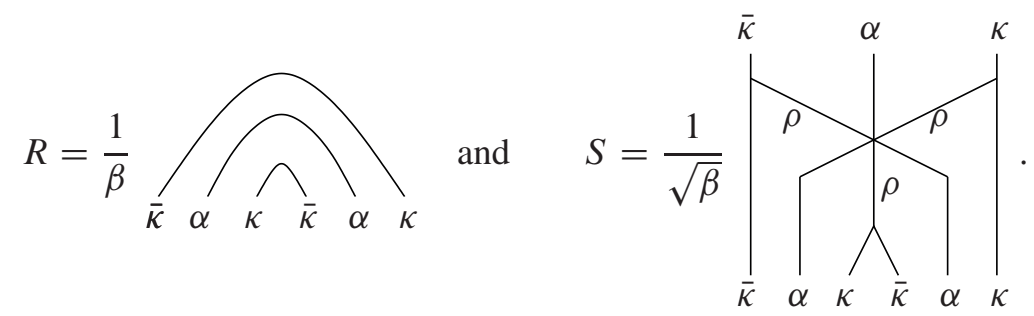

Lemma 4.2. Both $R$ and $S$ are isometries.

Proof. $R$ is clearly an isometry. We have 

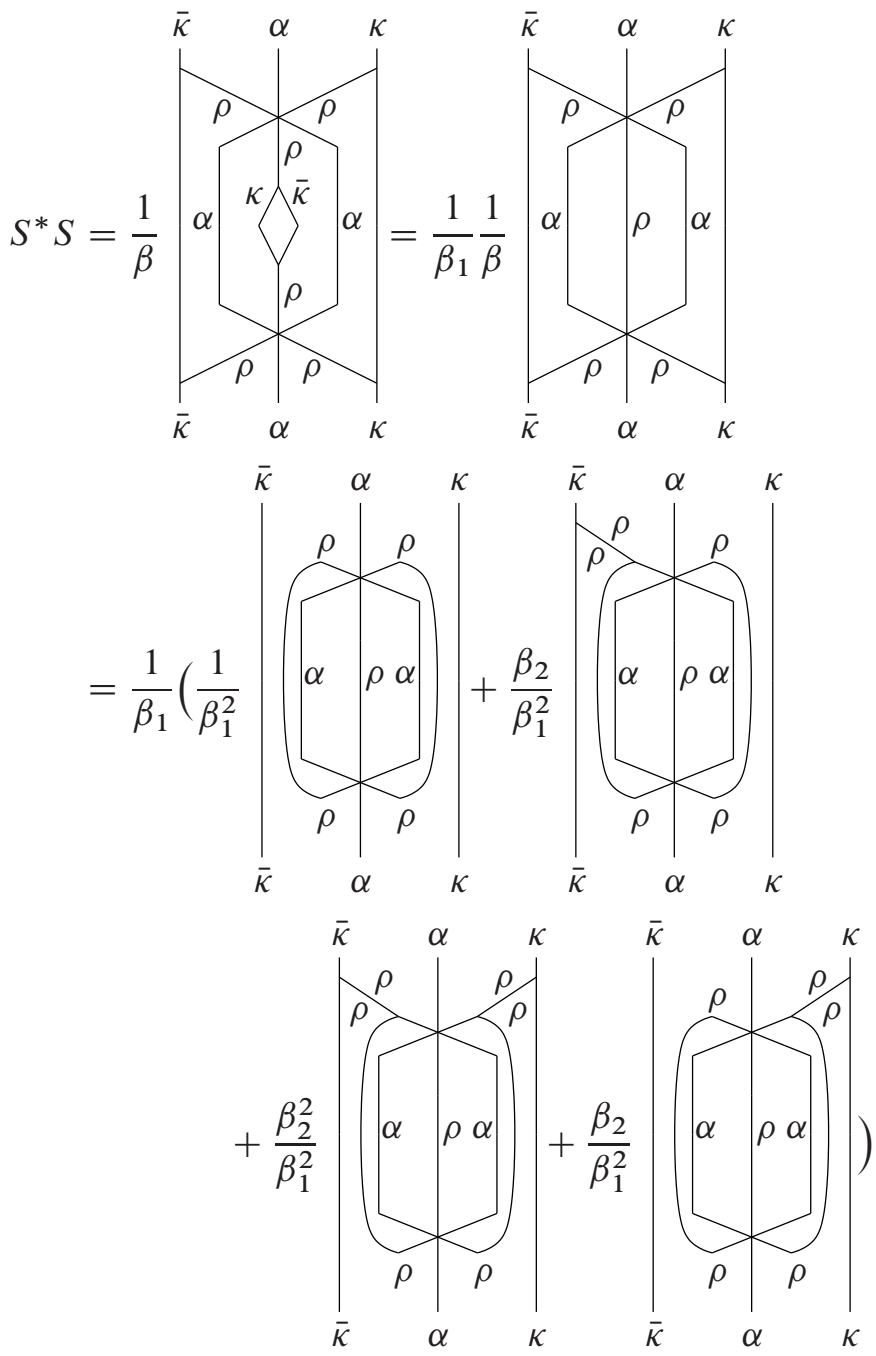

$=\frac{1}{\beta_{1}^{3}} \beta_{1}^{3} \operatorname{Id}_{\bar{\kappa} \alpha \kappa}=\operatorname{Id}_{\bar{\kappa} \alpha \kappa}$,

where we have used Lemma 4.1 twice to split the diagram into four, and Corollary 3.9 to evaluate the only nonzero term.

Lemma 4.3. We have $\left(S \otimes \operatorname{Id}_{\sigma}\right) \circ R=\left(\operatorname{Id}_{\sigma} \otimes S\right) \circ R$.

Proof. We have 


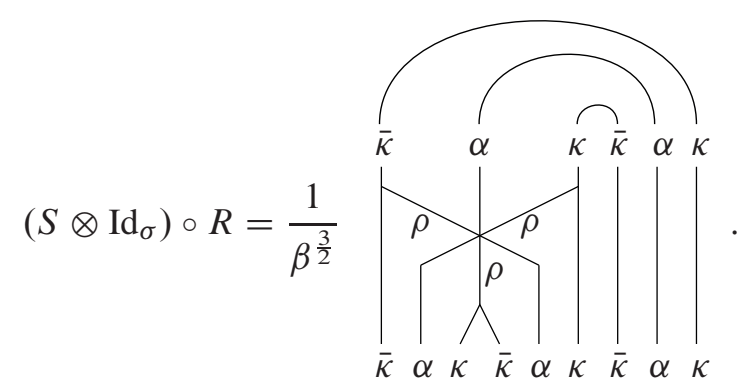

Its image under the linear isomorphism

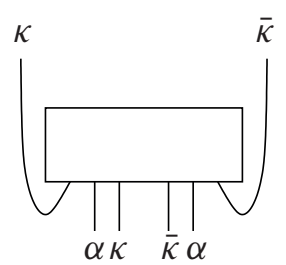

is

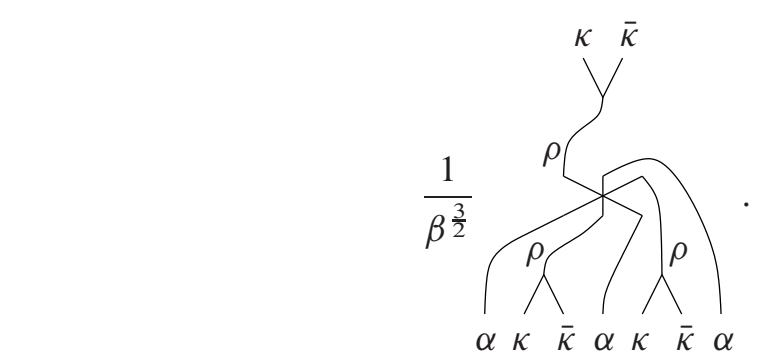

On the other hand,

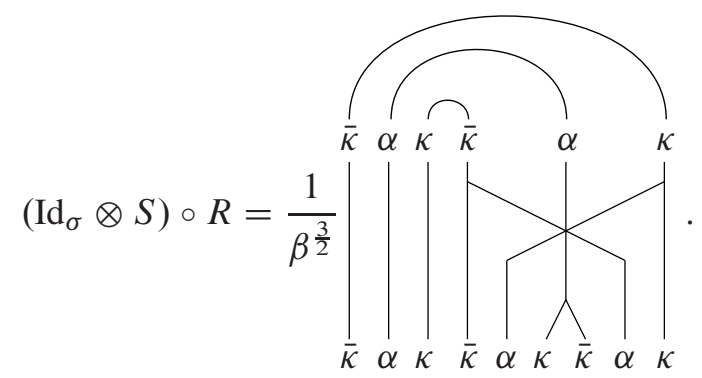

Its image under (3) is

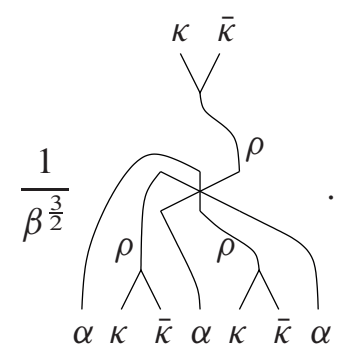


By Lemma 3.10 these are equal.

Lemma 4.4. We have

$$
\frac{\beta}{\beta_{1}^{2}}\left(R \otimes \operatorname{Id}_{\sigma}-\operatorname{Id}_{\sigma} \otimes R\right)=\left(\operatorname{Id}_{\sigma} \otimes S\right) \circ S-\left(S \otimes \operatorname{Id}_{\sigma}\right) \circ S .
$$

Proof. We have

$$
\begin{aligned}
& \frac{\beta}{\beta_{1}^{2}}\left(R \otimes \operatorname{Id}_{\bar{\kappa} \alpha \kappa}-\operatorname{Id}_{\bar{\kappa} \alpha \kappa} \otimes R\right) \\
& =\frac{1}{\beta_{1}^{2}}
\end{aligned}
$$

Its image under

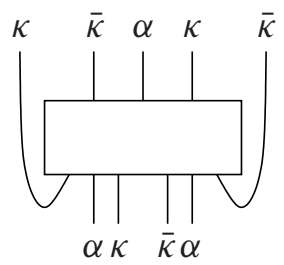

is

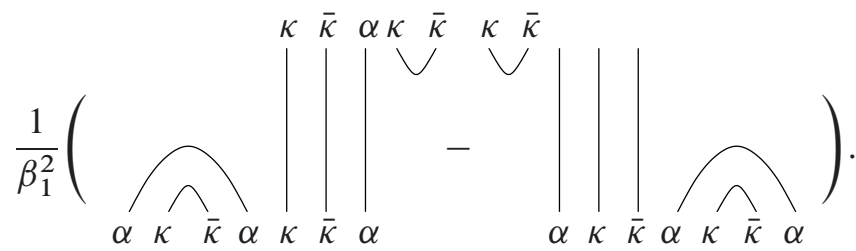

Using

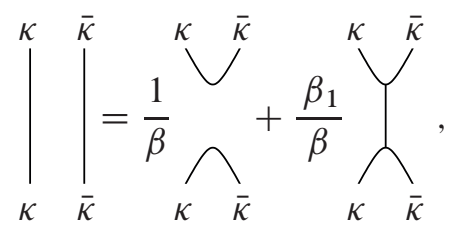

we get

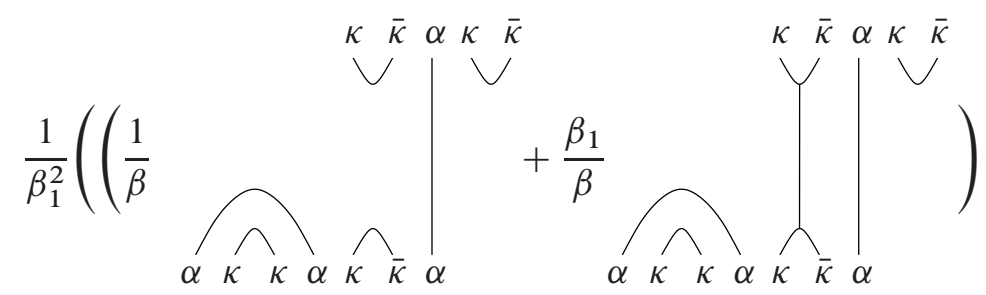


A quadrilateral in the Asaeda-Haagerup category

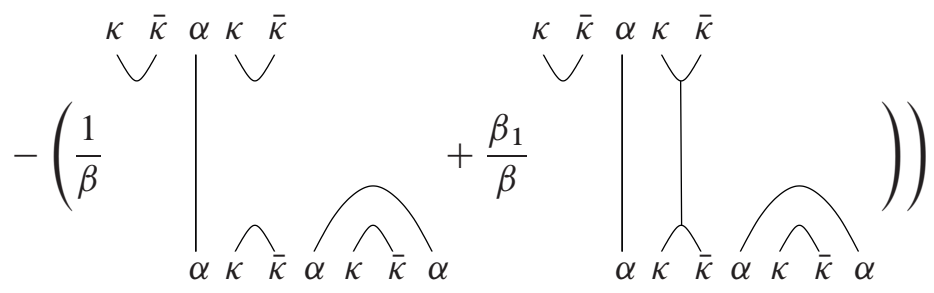

(by Lemma 3.12)

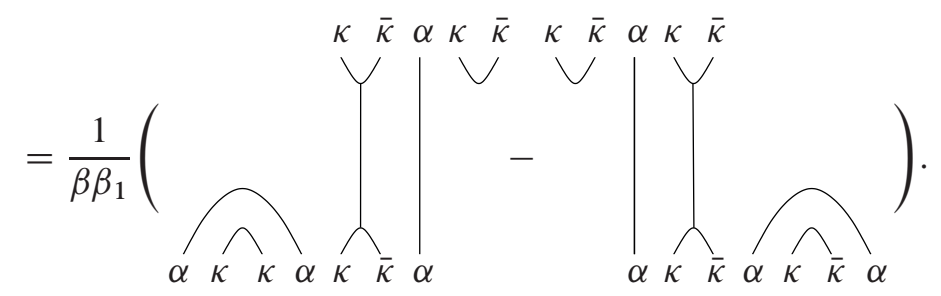

On the other hand,

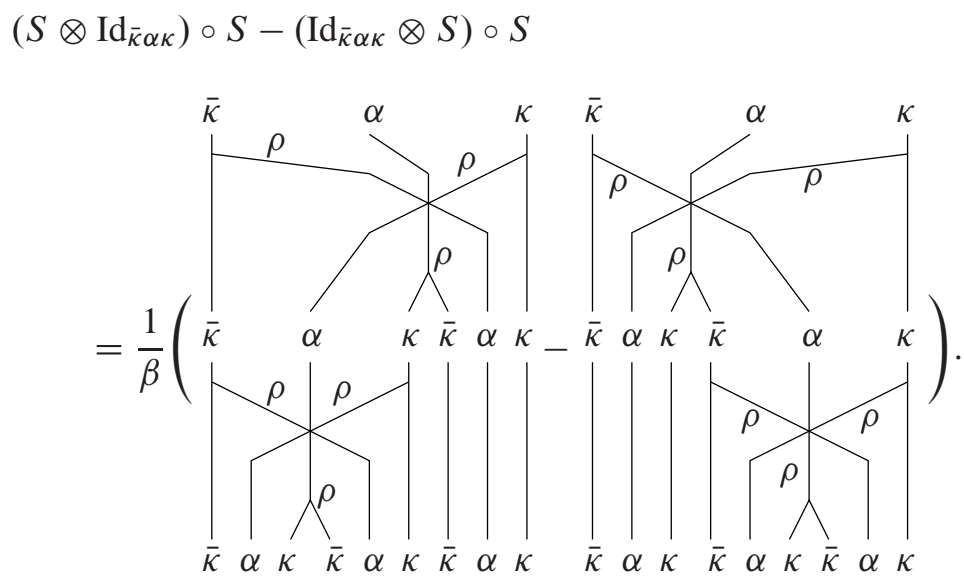

Its image under (4) is

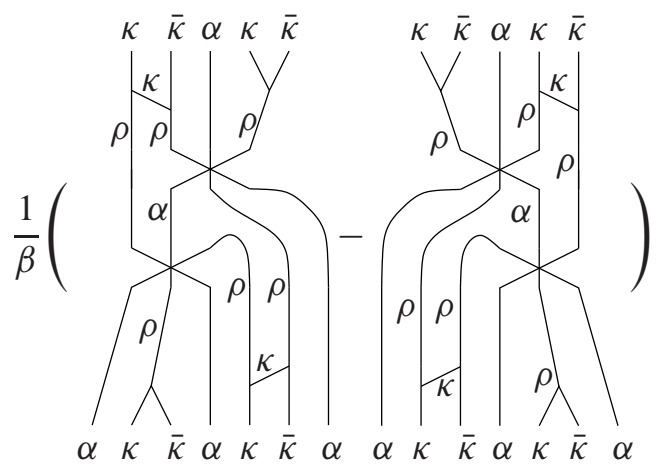


M. Asaeda and P. Grossman

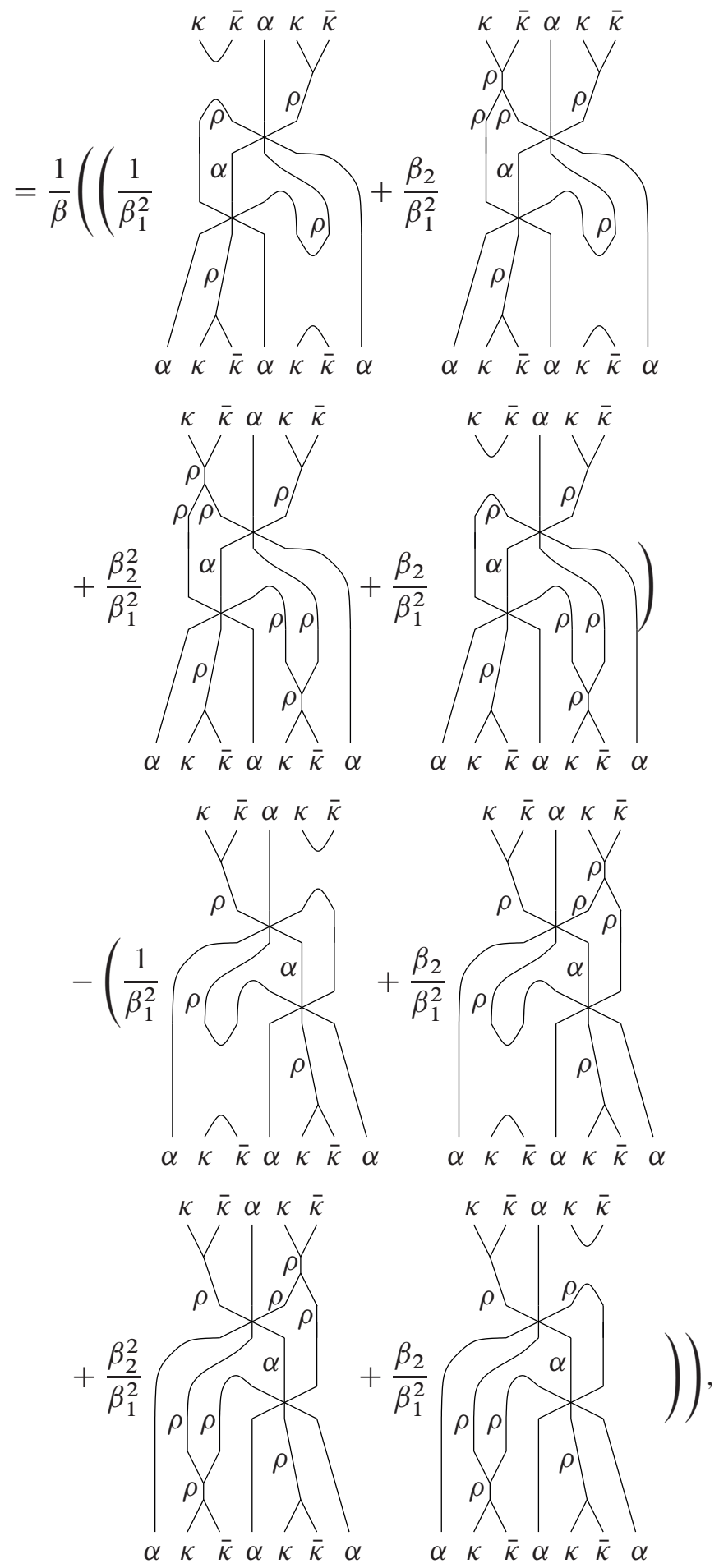


where we have used Lemma 4.1 twice on each side. Since $(\alpha \rho, \rho \alpha \rho)=(\rho \alpha, \rho \alpha \rho)=$ $(\alpha \rho, \alpha \rho \alpha \rho \alpha)=(\rho \alpha, \alpha \rho \alpha \rho \alpha)=0$, the second and fourth terms in each summand are 0 . Moreover, by Lemma 3.11 the third terms are the same so they cancel. That leaves

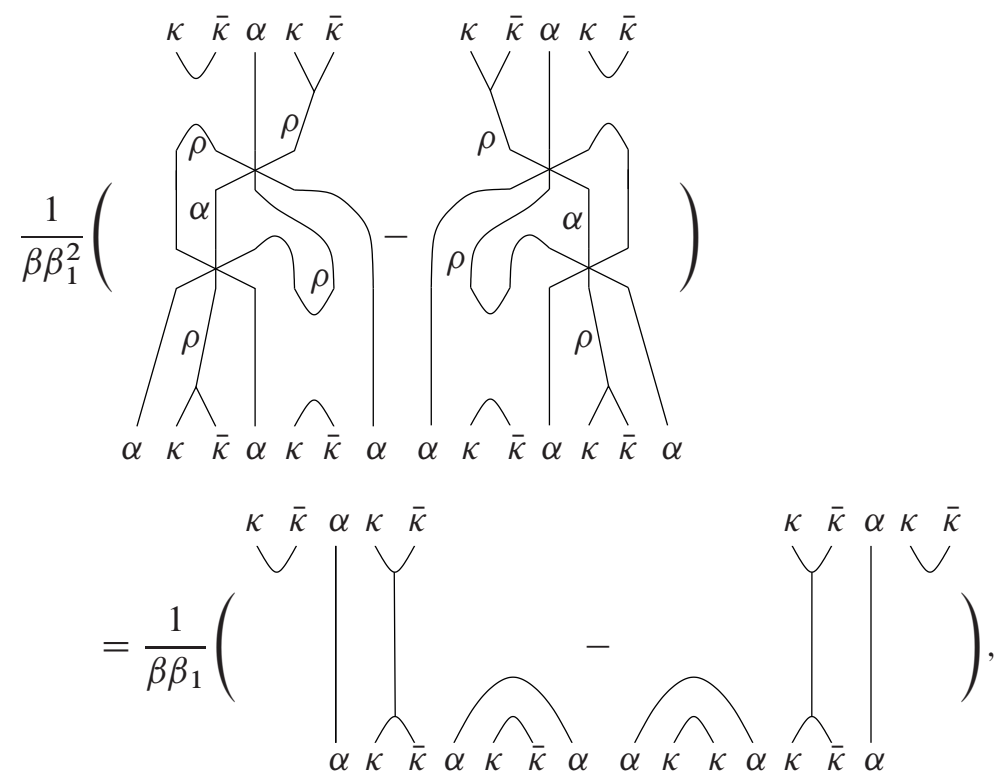

where we have used Lemma 3.8.

Theorem 4.5. The bimodule $\gamma=\operatorname{Id}_{M} \oplus \bar{\kappa} \alpha \kappa$ admits a $Q$-system, which is unique up to equivalence.

Proof. Existence is immediate from Lemmas 4.2, 4.3, 4.4 and Proposition 2.3. For uniqueness, note that, $\operatorname{since} \operatorname{dim}(\kappa \alpha \bar{\kappa}, \kappa \alpha \bar{\kappa} \kappa \alpha \bar{\kappa})=1, S$ is determined up to a scalar. For the equation in Lemma 4.4 to hold, that scalar is determined up to a sign, which means the $Q$-system is determined up to equivalence (see [5], Lemma 3.5).

Once existence of the $Q$-system is known, the principal graph of the corresponding subfactor can be easily computed from the Asaeda-Haagerup fusion rules:

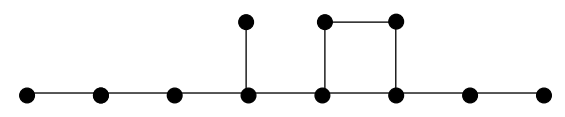

The dual graph was computed using the subfactor atlas and sent to the authors by Noah Snyder. It is

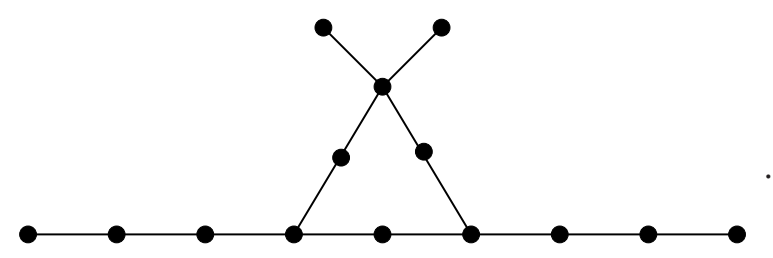


Note that the dual graph possesses an order two symmetry very similar to that of the original graph. We therefore conjecture that our construction may be iterated once more to obtain a $Q$-system in the Asaeda-Haagerup category with index $\frac{9+\sqrt{17}}{2}$. Checking this should be a straightforward computation, but we would first need some data from an analogue of Asaeda and Haagerup's original computation, applied to the new "AH+1" subfactor.

Theorem 4.6. There is an irreducible, noncommuting but cocommuting quadrilateral whose upper sides are the Asaeda-Haagerup subfactor. Such a quadrilateral is unique up to isomorphism of the planar algebra.

Proof. Let $P \subset M$ be a dual Asaeda-Haagerup subfactor with $\bar{\kappa}={ }_{P} M_{M}$ corresponding to the fundamental vertex on the dual Asaeda-Haagerup principal graph. By Theorem 4.5, we can find a subfactor $N \subset P$ such that $\bar{\iota} \cong \operatorname{Id}_{P} \oplus \bar{\kappa} \alpha \kappa$, where $\iota=$ ${ }_{N} P_{P}$. Then ${ }_{N} M_{M}=\iota \bar{\kappa}$, and $\operatorname{dim}(\iota \bar{\kappa}, \iota \bar{\kappa})=\operatorname{dim}(\bar{\iota}, \bar{\kappa} \kappa)=\operatorname{dim}\left(\operatorname{Id}_{P} \oplus \bar{\kappa} \alpha \kappa, \bar{\kappa} \kappa\right)=1$, so $N \subset M$ is irreducible.

By a slight abuse of notation, we will let $\alpha$ denote both the $(M-M)$-bimodule of dimension 1 and the corresponding outer automorphism of $M$. We have $\operatorname{dim}(\kappa \bar{\iota} \iota \bar{\kappa}, \alpha)=$ $\operatorname{dim}(\bar{\imath}, \bar{\kappa} \alpha \kappa)$, so $M_{M} M_{1 M}$ contains a copy of $[\alpha]$, where $N \subset M \subset M_{1}$ is the basic construction. Take a representative of $[\alpha]$ in the Galois group of $N \subset M$ and then set $Q=\alpha(P)$, and consider the quadrilateral

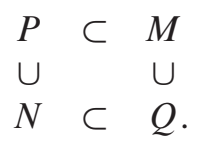

Then $P \neq Q$, or else either $N \subset P$ or $P \subset M$ would have a nontrivial Galois group, which is not the case. Since ${ }_{N} P_{N} \cong{ }_{N} \alpha(P)_{N}={ }_{N} Q_{N}$, the quadrilateral does not commute (see [5], Theorem 3.10). On the other hand, if $P \subset M \subset \bar{P}$ and $Q \subset M \subset \bar{Q}$ are each the basic construction, then ${ }_{M} L^{2}(\bar{P})_{M} \cong \kappa \bar{\kappa} \cong \operatorname{Id}_{M} \oplus \rho \not$ $\operatorname{Id}_{M} \oplus \alpha \rho \alpha \cong \alpha \kappa \bar{\kappa} \alpha \cong{ }_{M} L^{2}(\bar{Q})_{M}$. By [6], Lemma 4.2.1, the quadrilateral cocommutes. Uniqueness follows from [5], Theorem 4.8.

This theorem answers the conjecture in [5], Remark 5.16.

Remark 4.7. If the iterated equations hold and the $Q$-system for index $\frac{9+\sqrt{17}}{2}$ exists, there would similarly be a noncommuting quadrilateral whose upper sides are the “AH+1" subfactor and whose lower sides have index $\frac{9+\sqrt{17}}{2}$. 


\section{References}

[1] M. Asaeda and U. Haagerup, Exotic subfactors of finite depth with Jones indices $(5+\sqrt{13}) / 2$ and $(5+\sqrt{17}) / 2$. Commun. Math. Phys. 202 (1999), 1-63. MR 1686551 Zbl 1014.46042

[2] S. Bigelow, S. Morrison, E. Peters, and N. Snyder, Constructing the extended Haagerup planar algebra. Preprit 2009. arXiv:0909.4099. To appear in Acta Math.

[3] D. Bisch and V. F. R. Jones, Algebras associated to intermediate subfactors. Invent. Math. 128 (1997), 89-157. MR 1437496 Zbl 0891.46035

[4] D. E. Evans and Y. Kawahigashi, Quantum symmetries on operator algebras. Clarendon Press, Oxford, 1998. MR 1642584 Zbl 0924.46054

[5] P. Grossman and M. Izumi, Classification of noncommuting quadrilaterals of factors. Internat. J. Math. 19 (2008), 557-643. MR 2418197 Zbl 1153.46037

[6] P. Grossman, and V. F. R. Jones, Intermediate subfactors with no extra structure. J. Amer. Math. Soc. 20 (2007), 219-265. MR 2257402 Zbl 1131.46041

[7] U. Haagerup, Principal graphs of subfactors in the index range $4<3+\sqrt{2}$. In H. Araki (ed.) et al., Subfactors. Proceedings of the Taniguchi symposium on operator algebras, Kyuzeso, Japan, July 6-10, 1993. World Scientific, Singapore, 1994, 1-38. MR 1317352 Zbl 0933.46058

[8] M. Izumi, The structure of sectors associated with Longo-Rehren inclusions II. Examples. Rev. Math. Phys. 13 (2001), 603-674. MR 1832764 Zbl 1033.46506

[9] M. Izumi and H. Kosaki, On a subfactor analogue of the second cohomology. Rev. Math. Phys. 14 (2002), 733-757. MR 1932664 Zbl 1030.46098

[10] V. F. R. Jones, Index for subfactors. Invent. Math. 72 (1983), 1-25. MR 0696688 Zbl 0508.46040

[11] V. F. R. Jones, The planar algebra of a bipartite graph. In C. McA. Gordon (ed.) et al., Knots in Hellas '98 (Delphi), 94-117. World Sci. Publ., River Edge, NJ, 2000. http://math.berkeley.edu/ vfr/delphi.ps MR 1865703 Zbl 1021.46047

[12] R. Longo, A duality for Hopf algebras and for subfactors I. Comm. Math. Phys. 159 (1994), 133-150. MR 1257245 Zbl 0802.46075

[13] R. Longo and J. E. Roberts, A theory of dimension. K-theory, 11 (1997), 103-159. MR 1444286 Zbl 0874.18005

[14] T. Masuda, An analogue of Longo's canonical endomorphism for bimodule theory and its application to asymptotic inclusions. Internat. J. Math. 8 (1997), 249-265. MR 1442437 Zbl 0909.46044

[15] S. Popa, Classification of amenable subfactors of type II. Acta Math. 172 (1994), 163255. MR 1278111 Zbl 0853.46059

[16] T. Sano and Y. Watatani, Angles between two subfactors. J. Oper. Theory 32 (1994), 209-241. MR 1338739 Zbl 0838.46052

[17] Y. Watatani, Lattices of intermediate subfactors. J. Funct. Anal. 140 (1996), 312-334. MR 1409040 Zbl 0899.46050 
[18] F. Xu, On representing some lattices as lattices of intermediate subfactors of finite index. Adv. Math. 220 (2009), 1317-1356. MR 2493615 Zbl 1169.46031

Received August 11, 2010

Marta Asaeda, Department of Mathematics, University of California, Riverside, 900 University Ave., Riverside, CA 92521, U.S.A.

E-mail: marta@math.ucr.edu

Pinhas Grossman, School of Mathematics, Cardiff University, Senghennydd Road, Cardiff, Wales, CF24 4AG, U.K.

E-mail: pinhas@impa.br 